\title{
Assessment and Estimation of the Coefficients of a Linear Model for Interval Data
}

\author{
Amir Massoud Malekfar and Farzad Eskandari* \\ Allameh Tabataba'i University
}

Received: 1/14/2018 Approved: 11/5/2019

\begin{abstract}
Imprecise measurement tools produce imprecise data. Interval valued (interval) data is one type of data which is usually used to deal with such imprecision. So, interval-valued variables have been used in the last decade. The relationships between the variables have recently been modeled by linear regression models. If interval response variables have any statistical distributions, the relationships are modeled in the linear models framework. In this paper, we propose new estimators for the parameters of an interval linear model under some conditions. Under the conditions, we demonstrate the theoretical adequacy of the estimators. Simulation studies and a real-life case study show the empirical adequacy and the practical applicability of the new estimators, respectively, under the conditions.
\end{abstract}

Keywords. Interval-valued data, interval linear model, the theoretical and empirical adequacy of the estimators.

MSC 2010: 57R19.

\section{Introduction}

Imprecise measurement tools are imprecise-valued data (variably termed as single-valued data or classical data or real-valued data) producers. Diday

\footnotetext{
* Corresponding author

Copyright 10 2019, ASP Ins. This open-access article is published under the terms of the Creative Commons AttributionNonCommercial 4.0 International License which permits Share (copy and redistribute the material in any medium or format) and Adapt (remix, transform, and build upon the material) under the Attribution-NonCommercial terms.
} 
(1995), Émilion (1997), Bertrand and Goupil (2000), Afonso et al. (2007), and Kim and Billard (2011) proposed some methods for analyzing and modeling imprecise-valued data. Imprecise-valued data and a desire for increasing the precision of data are two motivations for statisticians to use intervalvalued (interval) data rather than single-valued data. Interval-valued data have been used to collect single-valued data (see for example Diday and Émilion, 1998). In recent years, big data and grouped data have been shown using interval-valued data (see for example Calle and Gómez, 2001; Rivero and Valdes, 2008; Trutschnig et al., 2009; Huber et al., 2009; Billard, 2011).

Different analyzing and modeling methods of interval-valued data have recently been proposed as follows: Billard and Diday (2000) introduced a linear regression model on centers of intervals. Hence, when this method is used, high volumes of information are lost. This method was called the center method (CM). Neto et al. (2004) and Neto and de Carvalho (2008) introduced the center and range method (CRM). The method utilizes not only centers but also ranges of intervals to fit regression models. Centers and ranges are used separately to do the fitting. Neto et al. (2005) and Neto and de Carvalho (2010) proposed the constrained center method (CCM). This method is the center method under a restrictive condition. Neto et al. (2005) and Neto and de Carvalho (2010) proposed the constrained center and range method (CCRM). The method is the center and range method under two restrictive conditions. The CRM assumes that centers and ranges are independent and fits models on them separately. In order to break the assumption, Afonso et al. (2007) fitted centers and ranges simultaneously as a bivariate model, either with (BCRMI) or without (BCRMO) interaction terms between the center and range variables. Blanco-Fernández et al. (2011) presented and estimated a simple linear model between random interval-valued variables. The complete information method (CIM) was introduced by Wang et al. (2012). This method transforms the regression modeling into the computation of some inner products of interval-valued variables.

Bertrand and Goupil (2000) introduced the sample mean and variance of interval data. Kim and Billard (2011) proposed the sample mean and variance of histogram-valued data. The sample covariance between interval data was obtained by Billard (2007). Le-Rademacher and Billard (2012) completed this method. Billard (2007), Gil et al. (2007), and Le-Rademacher and Billard (2012) analyzed dependence and independence of interval-valued data. 
Linear regression models (LRMs) have been used for modeling the relationships between interval variables in many recent articles. The relationships are modeled in the framework of linear models (LMs) if interval-valued response variables have any statistical distributions. In Blanco-Fernández et al. (2011), an interval linear model has been formalized based on the natural set-valued arithmetic and the associated least-square (LS) estimators have been determined. In the present work, we develop the approach applied by Blanco-Fernández et al. (2011) for LMs. In this paper, the estimators provided in Blanco-Fernández et al. (2011) are called the old known estimators. Based on a new structure of Monte Carlo resampling (MCR), this study proposes a set of new consistent estimators for the set of the parameters of the interval linear model provided in Blanco-Fernández et al. (2011) under some conditions.

Under these conditions, the new structure of MCR generates a large number of single-valued sample sets, each of which consists of single-valued points randomly chosen within random intervals, from the interval-valued variables of each $\mathrm{LM}$ with the structure of the interval linear model. We provide the new and old known estimators on the basis of the new structure of MCR.

In this paper, both the theoretical and the empirical adequacy of the new and old known estimators of the interval linear model are demonstrated under the conditions. This research shows that for some LMs with the structure of the interval linear model, the set of the new estimators under the conditions is superior to the set of the old known estimators.

The rest of the paper consists of six sections. Section 2 provides some preliminary concepts in the interval setting, and introduces the interval linear model presented in Blanco-Fernández et al. (2011). The new structure of MCR is defined in Section 3. Section 4 presents the old known and new estimators of the parameters of the interval linear model under the conditions. Section 5 studies and compares some optimal properties of the estimators under the conditions. The empirical performance of the estimators under the conditions is tested by means of some simulation studies in Section 6 . In this section, the superiority of the set of the new estimators to the set of the old known estimators is investigated. Moreover, in Section 6.2, we compare the practical applicability of the sets of the new and old known estimators by means of a real-life case study. The paper ends with some conclusions in Section 7 . 


\section{Introductory Concepts and an Interval Linear Model}

\subsection{Introductory Concepts of the Interval Framework}

The set of all closed real intervals is denoted as $\mathcal{K}_{c}(\mathbb{R})=\{[a, b]: a, b \in$ $\mathbb{R}, a \leqslant b\}$, where $c=\left\{(a, b) \in \mathbb{R}^{2} \mid a \leqslant b\right\}$. Each interval $N \in \mathcal{K}_{c}(\mathbb{R})$ can be written as $N=[\inf N, \sup N]$, where $\inf N, \sup N \in \mathbb{R}$ and $\inf N \leqslant$ $\sup N$. The interval $N$ can also be written as $N=[\operatorname{mid} N \pm \operatorname{spr} N]=$ $\left[\frac{\inf N+\sup N}{2} \pm \frac{\sup N-\inf N}{2}\right]$, where $\operatorname{mid} N=\frac{\inf N+\sup N}{2} \in \mathbb{R}$ and $\operatorname{spr} N=$ $\frac{\sup N-\inf N}{2} \in \mathbb{R}^{+}$denote the center (or midpoint or location) and the radius (or spread) of $N$, respectively. Also, $\operatorname{spr} N$ denotes the imprecision or the difference with a precise quantity of $\mathbb{R}$. In this paper, the (mid, spr)parametrization for interval-valued data is used.

Remark 1. The (inf, sup)-representation is not easy to use for statistical purposes given the order restriction that it involves. In this sense, it is more advisable to use the (mid, spr)-representation, since it only involves a nonnegativity constraint on the second component, which is more operative.

The linear model presented in Blanco-Fernández et al. (2011) is based on the (mid, spr)-characterization of the intervals. The notation $A=[\operatorname{mid} A \pm$ $\operatorname{spr} A]$ can be split into two terms depending on the midpoint and spread values of $A$ by means of the canonical decomposition $A=\operatorname{mid} A[1 \pm 0]+$ $\operatorname{spr} A[0 \pm 1]$. This expression allows us to work separately with the mid and spr components of the interval, but keeping the interval arithmetic. The intervals [1 $1 \pm 0],[0 \pm 1], \operatorname{mid} A[1 \pm 0], \operatorname{spr} A[0 \pm 1]$, and $A=\operatorname{mid} A[1 \pm 0]+$ $\operatorname{spr} A[0 \pm 1]$ can be equivalently expressed in their (inf, sup)-representation as $[1,1],[-1,1], \operatorname{mid} A[1,1]=[\operatorname{mid} A, \operatorname{mid} A], \operatorname{spr} A[-1,1]=[-\operatorname{spr} A, \operatorname{spr} A]$, and $A=[\operatorname{mid} A, \operatorname{mid} A]+[-\operatorname{spr} A, \operatorname{spr} A]=[\operatorname{mid} A-\operatorname{spr} A, \operatorname{mid} A+\operatorname{spr} A]$, respectively. For instance, we can write $[2,2]=2[1,1]=2[1 \pm 0],[-7,7]=$ $7[-1,1]=7[0 \pm 1]$, and $[-5,9]=[2 \pm 7]=[2-7,2+7]=[2,2]+[-7,7]=$ $2[1 \pm 0]+7[0 \pm 1]$. The expression $E([\operatorname{mid} B \pm \operatorname{spr} B])=[E(\operatorname{mid} B) \pm E(\operatorname{spr} B)]$ denotes the expected value of any random interval $B$ in terms of the Aumann expectation, whenever mid $B, \operatorname{spr} B \in L^{1}$.

In order to measure the distance between two intervals, an $L_{2}$-type metric has been exhaustively used and shown to be suitable on the space $\mathcal{K}_{c}(\mathbb{R})$. For every $A, Q \in \mathcal{K}_{c}(\mathbb{R})$, the $d$-distance is presented as 


$$
d(A, Q)=\sqrt{(\operatorname{mid} A-\operatorname{mid} Q)^{2}+(\operatorname{spr} A-\operatorname{spr} Q)^{2}} .
$$

Let $\mathcal{B}_{d}$ be the $\sigma$-field generated by the topology induced by $d$ on $\mathcal{K}_{c}(\mathbb{R})$. Let $(\Omega, \mathcal{A}, P)$ be a probability space. An interval-valued random variable $X=$ $[\inf X, \sup X]=[\operatorname{mid} X \pm \operatorname{spr} X]$ is a $\mathcal{B}_{d} \mid \mathcal{A}$-measurable function $X: \Omega \longrightarrow \mathcal{K}_{c}(\mathbb{R})$. Equivalently, $\inf X, \sup X, \operatorname{mid} X, \operatorname{spr} X: \Omega \rightarrow \mathbb{R}$, being real-valued random variables and $\operatorname{spr} X \geqslant 0$ almost sure with respect to probability $P$. The interval-valued variable $X$ can not be a single-valued (or a real-valued) variable with respect to the non-degenerated variable $\operatorname{spr} X$. The variance of the interval variable $X$ with respect to $E(X)=[E(\operatorname{mid} X) \pm E(\operatorname{spr} X)]$ in the metric space $\left(\mathcal{K}_{c}(\mathbb{R}), d\right)$ is expressed as

$$
\sigma^{2}(X)=E\left(d^{2}(X, E(X))\right)=\sigma^{2}(\operatorname{mid} X)+\sigma^{2}(\operatorname{spr} X),
$$

whenever $0<\sigma^{2}(\operatorname{mid} X), \sigma^{2}(\operatorname{spr} X)<\infty$. The variable $X=\operatorname{mid} X[1 \pm 0]+$ $\operatorname{spr} X[0 \pm 1]$ can be presented as $X=X^{M}+X^{S}$, where $X^{M}=\operatorname{mid} X[1 \pm 0]$ and $X^{S}=\operatorname{spr} X[0 \pm 1]$. Therefore, the expressions $\sigma^{2}\left(X^{M}\right)=\sigma^{2}(\operatorname{mid} X[1 \pm$ $0])=\sigma^{2}(\operatorname{mid} X[1,1])=[1,1] \sigma^{2}(\operatorname{mid} X)=\sigma^{2}(\operatorname{mid} X)$ and $\sigma^{2}\left(X^{S}\right)=\sigma^{2}(\operatorname{spr}$ $X[0 \pm 1])=\sigma^{2}(\operatorname{spr} X[-1,1])=[1,1] \sigma^{2}(\operatorname{spr} X)=\sigma^{2}(\operatorname{spr} X)$ can be defined. Hence, we can express $\sigma^{2}(X)=\sigma^{2}\left(X^{M}\right)+\sigma^{2}\left(X^{S}\right)$. The expression $\sigma(X, Y)$ is often defined as the corresponding $d$-covariance in $\mathbb{R}^{2}$ through the (mid, spr)-parametrization of the intervals, leading to the expression

$$
\begin{aligned}
\sigma(X, Y)= & \sigma(\operatorname{mid} X, \operatorname{mid} Y)+\sigma(\operatorname{spr} X, \operatorname{spr} Y) \\
= & E[(\operatorname{mid} X-E(\operatorname{mid} X))(\operatorname{mid} Y-E(\operatorname{mid} Y))] \\
& +E[(\operatorname{spr} X-E(\operatorname{spr} X))(\operatorname{spr} Y-E(\operatorname{spr} Y))],
\end{aligned}
$$

whenever $\sigma(\operatorname{mid} X, \operatorname{mid} Y), \sigma(\operatorname{spr} X, \operatorname{spr} Y)<\infty$. We can also write

$$
\begin{aligned}
\sigma\left(X^{M}, Y^{M}\right) & =\sigma(\operatorname{mid} X[1,1], \operatorname{mid} Y[1,1])=[1,1] \sigma(\operatorname{mid} X, \operatorname{mid} Y) \\
& =\sigma(\operatorname{mid} X, \operatorname{mid} Y)
\end{aligned}
$$

$$
\begin{aligned}
\sigma\left(X^{S}, Y^{S}\right) & =\sigma(\operatorname{spr} X[-1,1], \operatorname{spr} Y[-1,1]=[-1,1][-1,1] \sigma(\operatorname{spr} X, \operatorname{spr} Y) \\
& =[1,1] \sigma(\operatorname{spr} X, \operatorname{spr} Y)=\sigma(\operatorname{spr} X, \operatorname{spr} Y)
\end{aligned}
$$

and hence $\sigma(X, Y)=\sigma\left(X^{M}, Y^{M}\right)+\sigma\left(X^{S}, Y^{S}\right)$. 


\subsection{An Interval Linear Model}

Let $X$ and $Y$ be two random intervals with finite second-order moments, and spr $X$ be non-degenerated. The linear model proposed in Blanco-Fernández et al. (2011) between the interval-valued random independent and response variable $X$ and $Y$, respectively, is formalized on the basis of the canonical decomposition as follows:

$$
Y=\alpha \operatorname{mid} X[1 \pm 0]+\beta \operatorname{spr} X[0 \pm 1]+\gamma[1 \pm 0]+\varepsilon,
$$

where $\alpha$ and $\beta$ are the coefficients of the linear model, $\gamma$ is an intercept term affecting the mid component of $Y$, and $\varepsilon$ is an interval-valued random error variable such that $E(\varepsilon \mid X)=[-\delta, \delta] \in \mathcal{K}_{c}(\mathbb{R})$, with $\delta \geqslant 0$. The interval linear model (1) induces linear relationships between the mid and the spr variables of intervals $X$ and $Y$. To be precise, mid $Y=\alpha \operatorname{mid} X+\gamma+\operatorname{mid} \varepsilon$ and $\operatorname{spr} Y=|\beta| \operatorname{spr} X+\operatorname{spr} \varepsilon$. By considering the definitions $X^{M}=\operatorname{mid} X[1 \pm 0]$ and $X^{S}=\operatorname{spr} X[0 \pm 1]$, the linear function associated with the interval linear model given in (1) is $E(Y \mid X)=\alpha X^{M}+\beta X^{S}+B$, where $B=[\gamma-\delta, \gamma+\delta] \epsilon$ $\mathcal{K}_{c}(\mathbb{R})$. By considering the random interval $X^{S}=\operatorname{spr} X[0 \pm 1]$, we can write $X^{S}=-X^{S}$. Hence, the linear model (1) can always be expressed as follows:

$$
Y=\alpha X^{M}+(-\beta) X^{S}+\gamma[1 \pm 0]+\varepsilon=\alpha X^{M}+\beta X^{S}+\gamma[1 \pm 0]+\varepsilon .
$$

Based on the model (2), we can always look for the non-negative estimate of the parameter $\beta$. The model (2) can be written as the sum of the separate models $Y^{M}=\alpha X^{M}+\gamma[1 \pm 0]+\varepsilon^{M}$ and $Y^{S}=\beta X^{S}+\varepsilon^{S}=(-\beta) X^{S}+\varepsilon^{S}$.

\section{A New Structure of MCR}

The introduction of a new structure of MCR is one of the innovations and novelties of this article. This approach is utilized to fit the model (1) on interval-valued data. The structure generates samples in each repetition by randomly selecting a single-valued point within each random interval from the interval-valued variables of the model (1). By the nature of Monte Carlo simulation (MCS), a larger number of repetitions is always desired. The structure is computationally intensive. One of the benefits of the structure is that it fully makes use of the variability of the interval-valued data. Based on this structure, a researcher selects single-valued samples within the random intervals of interval-valued variables. However, based on other sampling 
methods from interval variables, the researcher selects single-valued samples from mid variables and $s p r$ variables. Another benefit of the structure is that it relieves the need to develop complex methodologies for interval-valued data. The new structure of MCR is introduced in the rest of this section.

The $i$ th random sample from the single-valued variable mid $\varepsilon$ at the $b$ th iteration is defined as $\left\{\operatorname{mid} \varepsilon_{i}^{b}\right\}, i=1, \ldots, k$ and $b=1, \ldots, n$. We introduce $\left\{\varepsilon_{i}^{M b *}\right\}, i=1, \ldots, k$ and $b=1, \ldots, n$, as a real-valued random sample from the interval $\varepsilon_{i}^{M b}=\left[\operatorname{mid} \varepsilon_{i}^{b}, \operatorname{mid} \varepsilon_{i}^{b}\right]=\operatorname{mid} \varepsilon_{i}^{b}$ of the variable $\varepsilon^{M}=$ $\operatorname{mid} \varepsilon[1,1]=\operatorname{mid} \varepsilon$. Hence, $\varepsilon_{i}^{M b *}$ is the same $\operatorname{mid} \varepsilon_{i}^{b}$. The $b$ th, $b=1, \ldots, n$, random sample set from the interval variable $\varepsilon^{M}$ is

$$
\varepsilon^{M * b}=\left\{\varepsilon_{1}^{M b *}, \ldots, \varepsilon_{k}^{M b *}\right\} .
$$

The sample $\left\{\operatorname{spr} \varepsilon_{i}^{b}\right\}$ is introduced as the $i$ th real-valued random sample, $i=1, \ldots, k$ and $b=1, \ldots, n$, obtained from the single-valued variable $\operatorname{spr} \varepsilon$ in the $b$ th repetition. Hence, the $b$ th $, b=1, \ldots, n$, random sample set from the variable $\operatorname{spr} \varepsilon$ is defined as

$$
\varepsilon^{S p * b}=\left\{\operatorname{spr} \varepsilon_{1}^{b}, \ldots, \operatorname{spr} \varepsilon_{k}^{b}\right\} .
$$

The sample $\left\{\varepsilon_{i}^{S b *}\right\}, i=1, \ldots, k$ and $b=1, \ldots, n$, is defined as a singlevalued random sample from the interval $\varepsilon_{i}^{S b}=\left[-\operatorname{spr} \varepsilon_{i}^{b}, \operatorname{spr} \varepsilon_{i}^{b}\right]$ of the interval variable $\varepsilon^{S}=[-\operatorname{spr} \varepsilon, \operatorname{spr} \varepsilon]$. So,

$$
\varepsilon^{S * b}=\left\{\varepsilon_{1}^{S b *}, \ldots, \varepsilon_{k}^{S b *}\right\}
$$

is the $b$ th, $b=1, \ldots, n$, random sample set from the variable $\varepsilon^{S}$. In the model (1), $\varepsilon$ is an interval-valued random error variable. Based on the $i$ th element of the random sample sets $\varepsilon^{M * b}$ and $\varepsilon^{S * b}$, respectively, in (3) and (5), we generate the single-valued element $\varepsilon_{i}^{b *}=\varepsilon_{i}^{M b *}+\varepsilon_{i}^{S b *}$ from the interval $\varepsilon_{i}^{b}=\varepsilon_{i}^{M b}+\varepsilon_{i}^{S b}=\left[\operatorname{mid} \varepsilon_{i}^{b}-\operatorname{spr} \varepsilon_{i}^{b}, \operatorname{mid} \varepsilon_{i}^{b}+\operatorname{spr} \varepsilon_{i}^{b}\right]$ at the $b$ th iteration, $i=1, \ldots, k$ and $b=1, \ldots, n$. So, for $i=1, \ldots, k$ and $b=1, \ldots, n$, the sample $\left\{\varepsilon_{i}^{b *}\right\}$ is introduced as a random sample from the interval $\varepsilon_{i}^{b}$ of the variable $\varepsilon=\varepsilon^{M}+\varepsilon^{S}=\operatorname{mid} \varepsilon[1 \pm 0]+\operatorname{spr} \varepsilon[0 \pm 1]$. Hence,

$$
\varepsilon^{* b}=\left\{\varepsilon_{1}^{b *}, \ldots, \varepsilon_{k}^{b *}\right\}
$$

is defined as the $b$ th sample set from the interval variable $\varepsilon$. 
We define the $i$ th, $i=1, \ldots, k$, real-valued random sample from the variable $\operatorname{spr} X$ at the $b$ th iteration as $\left\{\operatorname{spr} X_{i}^{b}\right\}, b=1, \ldots, n$. Hence, the $b$ th, $b=1, \ldots, n$, random sample set from the variable $\operatorname{spr} X$ is provided as

$$
X^{S p * b}=\left\{\operatorname{spr} X_{1}^{b}, \ldots, \operatorname{spr} X_{k}^{b}\right\}
$$

A random sample from the interval $X_{i}^{S b}=\left[-\operatorname{spr} X_{i}^{b}, \operatorname{spr} X_{i}^{b}\right]$ of the interval variable $X^{S}=[-\operatorname{spr} X, \operatorname{spr} X]$ is introduced as $\left\{X_{i}^{S b *}\right\}, i=1, \ldots, k$ and $b=1, \ldots, n . \quad X_{i}^{S b *} \in X_{i}^{S b}$, for $i=1, \ldots, k$ and $b=1, \ldots, n$, is singlevalued. So, the $b$ th, $b=1, \ldots, n$, sample set from the variable $X^{S}$ is

$$
X^{S * b}=\left\{X_{1}^{S b *}, \ldots, X_{k}^{S b *}\right\} .
$$

The $i$ th real-valued random sample from the single-valued variable mid $X$ in the $b$ th repetition is provided as $\left\{\operatorname{mid} X_{i}^{b}\right\}, i=1, \ldots, k$ and $b=1, \ldots, n$. $\left\{X_{i}^{M b *}\right\}$ is introduced as a real-valued random sample from the interval $X_{i}^{M b}=\operatorname{mid} X_{i}^{b}[1,1]=\operatorname{mid} X_{i}^{b}$ of the variable $X^{M}=\operatorname{mid} X[1,1]=\operatorname{mid} X$. Hence, $X_{i}^{M b *} \in X_{i}^{M b}$ can be expressed as $X_{i}^{M b *}=X_{i}^{M b}=\operatorname{mid} X_{i}^{b}, i=$ $1, \ldots, k$ and $b=1, \ldots, n$. So,

$$
X^{M * b}=\left\{X_{1}^{M b *}, \ldots, X_{k}^{M b *}\right\}
$$

is the $b$ th, $b=1, \ldots, n$, random sample set from the variable $X^{M}$. The random sample $\left\{X_{i}^{b *}\right\}$ is defined as a real-valued random sample from the interval $X_{i}^{b}=X_{i}^{M b}+X_{i}^{S b}=\left[\operatorname{mid} X_{i}^{b}-\operatorname{spr} X_{i}^{b}\right.$, mid $\left.X_{i}^{b}+\operatorname{spr} X_{i}^{b}\right]$ (the $i$ th random interval from the variable $X=X^{M}+X^{S}$ at $b$ th iteration) of the interval variable $X, i=1, \ldots, k$ and $b=1, \ldots, n$. We can express $X_{i}^{b *} \in X_{i}^{b}$. So, we introduce the $b$ th $, b=1, \ldots, n$, random sample set from the intervals of the variable $X=[\operatorname{mid} X \pm \operatorname{spr} X]$ as

$$
X^{* b}=\left\{X_{1}^{b *}, \ldots, X_{k}^{b *}\right\} .
$$

We can express $X_{i}^{b *}=X_{i}^{M b *}+X_{i}^{S b *}, i=1, \ldots, k$ and $b=1, \ldots, n$, where $X_{i}^{b *}, X_{i}^{M b *}$, and $X_{i}^{S b *}$ are the $i$ th element of the sample sets $X^{* b}, X^{M * b}$, and $X^{S * b}$ given in (10)-(8), respectively.

The $i$ th random sample from the real-valued variable $\operatorname{mid} Y$ in the $b$ th repetition is introduced as $\left\{\operatorname{mid} Y_{i}^{b}\right\}, i=1, \ldots, k$ and $b=1, \ldots, n$. We define $\left\{Y_{i}^{M b *}\right\}$ as a real-valued sample from the interval $Y_{i}^{M b}=\operatorname{mid} Y_{i}^{b}[1,1]=$ 
$\operatorname{mid} Y_{i}^{b}$ of the variable $Y^{M}=\operatorname{mid} Y[1,1]=\operatorname{mid} Y$. Hence, $Y_{i}^{M b *} \in Y_{i}^{M b}$ is the same $Y_{i}^{M b *}=Y_{i}^{M b}=\operatorname{mid} Y_{i}^{b}, i=1, \ldots, k$ and $b=1, \ldots, n$. The $b$ th sample set from the variable $Y^{M}=\operatorname{mid} Y$ is provided as

$$
Y^{M * b}=\left\{Y_{1}^{M b *}, \ldots, Y_{k}^{M b *}\right\} .
$$

We introduce the $i$ th single-valued sample from the real-valued variable $\operatorname{spr} Y$ in the $b$ th repetition as $\left\{\operatorname{spr} Y_{i}^{b}\right\}, i=1, \ldots, k$ and $b=1, \ldots, n$. The $b$ th random sample set from the variable $\operatorname{spr} Y$ is defined as

$$
Y^{S p * b}=\left\{\operatorname{spr} Y_{1}^{b}, \ldots, \operatorname{spr} Y_{k}^{b}\right\}
$$

In the $b$ th repetition, the single-valued sample $\left\{Y_{i}^{S b *}\right\}, i=1, \ldots, k$ and $b=1, \ldots, n$, is provided from the interval $Y_{i}^{S b}=\left[-\operatorname{spr} Y_{i}^{b}, \operatorname{spr} Y_{i}^{b}\right]$ of the interval variable $Y^{S}=\operatorname{spr} Y[-1,1]$. The $b$ th sample set from the variable $Y^{S}$ is provided as

$$
Y^{S * b}=\left\{Y_{1}^{S b *}, \ldots, Y_{k}^{S b *}\right\}
$$

At the $b$ th iteration, a single-valued sample from the interval $Y_{i}^{b}=Y_{i}^{M b}+$ $Y_{i}^{S b}=\left[\operatorname{mid} Y_{i}^{b}-\operatorname{spr} Y_{i}^{b}, \operatorname{mid} Y_{i}^{b}+\operatorname{spr} Y_{i}^{b}\right]$ (the $i$ th random interval from the variable $Y=Y^{M}+Y^{S}$ in the $b$ th repetition) of the interval-valued variable $Y$ is defined as $\left\{Y_{i}^{b *}\right\}, i=1, \ldots, k$ and $b=1, \ldots, n$. The $b$ th random sample set from the variable $Y$ is presented as

$$
Y^{* b}=\left\{Y_{1}^{b *}, \ldots, Y_{k}^{b *}\right\} .
$$

We can express $Y_{i}^{b *}=Y_{i}^{M b *}+Y_{i}^{S b *}, i=1, \ldots, k$ and $b=1, \ldots, n$, where $Y_{i}^{b *}, Y_{i}^{M b *}$, and $Y_{i}^{S b *}$ are the $i$ th element of the sample sets $Y^{* b}, Y^{M * b}$, and $Y^{S * b}$ given in (14), (11), and (13), respectively.

Assume that $G$ is a real-valued variable such that $G \stackrel{\mathcal{L}}{\longrightarrow} \underline{0}$ (that is $E(G) \rightarrow 0$ and $\left.\sigma^{2}(G) \rightarrow 0\right)$. For instance, we can consider $G \sim \mathrm{U}(-0.05,0.05)$. We define the interval variable $Y^{I}$ as $Y^{I}=\left[\operatorname{mid} Y^{I}-\operatorname{spr} Y^{I}, \operatorname{mid} Y^{I}+\operatorname{spr} Y^{I}\right]$ $=[\operatorname{mid} Y-\operatorname{spr} Y-|G|, \operatorname{mid} Y+\operatorname{spr} Y+|G|]=\operatorname{mid} Y[1 \pm 0]+(\operatorname{spr} Y+|G|)$ $[0 \pm 1]$, where $\operatorname{mid} Y^{I}=\operatorname{mid} Y$ (hence, $\operatorname{mid} Y^{I}-\operatorname{mid} Y \stackrel{\mathcal{L}}{\longrightarrow} \underline{0}$ is always confirmed) and $\operatorname{spr} Y^{I}=\operatorname{spr} Y+|G|$. By considering $|G|[0 \pm 1]=G[0 \pm 1]=$ $-G[0 \pm 1]$, if we have $G \stackrel{\mathcal{L}}{\longrightarrow} \underline{0}$, then we will conclude $Y^{I}-Y \stackrel{\mathcal{L}}{\longrightarrow} \underline{0}$. This 
result will be used in all the sections of this paper. We introduce $\left\{G_{i}^{b *}\right\}$ as the $i$ th single-valued random sample from the variable $G$ in the $b$ th repetition, $i=1, \ldots, k$ and $b=1, \ldots, n$. Based on the sample sets $Y^{* b}, Y^{M * b}$, and $Y^{S p * b}$ in (14), (11), and (12), respectively, we generate the $i$ th singlevalued sample $\left\{Y_{i}^{I b *}\right\}, i=1, \ldots, k$ and $b=1, \ldots, n$, from the subinterval $\left[Y_{i}^{b *}-\left|G_{i}^{b *}\right|, Y_{i}^{b *}+\left|G_{i}^{b *}\right|\right]$ of the interval $\left[Y_{i}^{M b *}-\operatorname{spr} Y_{i}^{b}-\left|G_{i}^{b *}\right|, Y_{i}^{M b *}+\operatorname{spr}\right.$ $\left.Y_{i}^{b}+\left|G_{i}^{b *}\right|\right]$ of the variable $Y^{I}$ at the $b$ th iteration. Hence, the values $Y_{i}^{I b *}$ and $Y_{i}^{b *}$ are close to each other with this sampling method. The $b$ th random sample set from the variable $Y^{I}$ is defined as

$$
Y^{I * b}=\left\{Y_{1}^{I b *}, \ldots, Y_{k}^{I b *}\right\} .
$$

Based on the $i$ th element of the sample sets $Y^{S p * b}$ and $Y^{S * b}$ in (12) and (13), respectively, the $i$ th single-valued sample $\left\{Y_{i}^{I S b *}\right\}, i=1, \ldots, k$ and $b=$ $1, \ldots, n$, is generated from the subinterval $\left[Y_{i}^{S b *}-\left|G_{i}^{b *}\right|, Y_{i}^{S b *}+\left|G_{i}^{b *}\right|\right]$ of the interval $\left[-\operatorname{spr} Y_{i}^{b}-\left|G_{i}^{b *}\right|, \operatorname{spr} Y_{i}^{b}+\left|G_{i}^{b *}\right|\right]$ of the variable $Y^{I S}=\operatorname{spr} Y^{I}[-1$ $, 1]=(\operatorname{spr} Y+|G|)[-1,1]$ at the $b$ th iteration. The $b$ th sample set from the variable $Y^{I S}$ is

$$
Y^{I S * b}=\left\{Y_{1}^{I S b *}, \ldots, Y_{k}^{I S b *}\right\} .
$$

According to the $i$ th element of the set $Y^{M * b}$ in (11), we generate the $i$ th single-valued sample $\left\{Y_{i}^{I M b *}\right\}, i=1, \ldots, k$ and $b=1, \ldots, n$, from the interval $\left[Y_{i}^{M b *}, Y_{i}^{M b *}\right]$ of the variable $Y^{I M}=\operatorname{mid} Y^{I}[1,1]=\operatorname{mid} Y[1,1]=$ mid $Y$ at the $b$ th iteration. Hence, $Y_{i}^{I M b *}$ is equal to $Y_{i}^{M b *}$. The $b$ th random sample set from the variable $Y^{I M}$ is as

$$
Y^{I M * b}=\left\{Y_{1}^{I M b *}, \ldots, Y_{k}^{I M b *}\right\} .
$$

Also, based on the set (12), the bth sample set from the non-negative variable $\operatorname{spr} Y^{I}=\operatorname{spr} Y+|G|$ is provided as

$$
Y^{I S p * b}=\left\{\operatorname{spr} Y_{1}^{b}+\left|G_{1}^{b *}\right|, \ldots, \operatorname{spr} Y_{k}^{b}+\left|G_{k}^{b *}\right|\right\} .
$$

We can write $Y_{i}^{I b *}=Y_{i}^{I M b *}+Y_{i}^{I S b *}, i=1, \ldots, k$ and $b=1, \ldots, n$, where $Y_{i}^{I b *}, Y_{i}^{I S b *}$, and $Y_{i}^{I M b *}$ are the $i$ th element of the data sets $Y^{I * b}, Y^{I S * b}$, and $Y^{I M * b}$ in (15)-(17), respectively.

By considering all $n$ iterations, based on (3)-(18), respectively, the ran- 
dom sample sets $\varepsilon^{M *}, \varepsilon^{S p *}, \varepsilon^{S *}, \varepsilon^{*}, X^{S p *}, X^{S *}, X^{M *}, X^{*}, Y^{M *}, Y^{S p *}, Y^{S *}$, $Y^{*}, Y^{I *}, Y^{I S *}, Y^{I M *}$, and $Y^{I S p *}$ are produced as follows:

$$
\begin{gathered}
\varepsilon^{M *}=\left\{\varepsilon_{1}^{M 1 *}, \ldots, \varepsilon_{k}^{M 1 *}, \ldots, \varepsilon_{1}^{M n *}, \ldots, \varepsilon_{k}^{M n *}\right\}, \\
\varepsilon^{S p *}=\left\{\operatorname{spr} \varepsilon_{1}^{1}, \ldots, \operatorname{spr} \varepsilon_{k}^{1}, \ldots, \operatorname{spr} \varepsilon_{1}^{n}, \ldots, \operatorname{spr} \varepsilon_{k}^{n}\right\}, \\
\varepsilon^{S *}=\left\{\varepsilon_{1}^{S 1 *}, \ldots, \varepsilon_{k}^{S 1 *}, \ldots, \varepsilon_{1}^{S n *}, \ldots, \varepsilon_{k}^{S n *}\right\}, \\
\varepsilon^{*}=\left\{\varepsilon_{1}^{1 *}, \ldots, \varepsilon_{k}^{1 *}, \ldots, \varepsilon_{1}^{n *}, \ldots, \varepsilon_{k}^{n *}\right\}, \\
X^{S p *}=\left\{\operatorname{spr} X_{1}^{1}, \ldots, \operatorname{spr} X_{k}^{1}, \ldots, \operatorname{spr} X_{1}^{n}, \ldots, \operatorname{spr} X_{k}^{n}\right\}, \\
X^{S *}=\left\{X_{1}^{S 1 *}, \ldots, X_{k}^{S 1 *}, \ldots, X_{1}^{S n *}, \ldots, X_{k}^{S n *}\right\}, \\
X^{M *}=\left\{X_{1}^{M 1 *}, \ldots, X_{k}^{M 1 *}, \ldots, X_{1}^{M n *}, \ldots, X_{k}^{M n *}\right\}, \\
X^{*}=\left\{X_{1}^{1 *}, \ldots, X_{k}^{1 *}, \ldots, X_{1}^{n *}, \ldots, X_{k}^{n *}\right\}, \\
Y^{M *}=\left\{Y_{1}^{M 1 *}, \ldots, Y_{k}^{M 1 *}, \ldots, Y_{1}^{M n *}, \ldots, Y_{k}^{M n *}\right\}, \\
Y^{S p *}=\left\{\operatorname{spr} Y_{1}^{1}, \ldots, \operatorname{spr} Y_{k}^{1}, \ldots, \operatorname{spr} Y_{1}^{n}, \ldots, \operatorname{spr} Y_{k}^{n}\right\}, \\
Y^{S *}=\left\{Y_{1}^{S 1 *}, \ldots, Y_{k}^{S 1 *}, \ldots, Y_{1}^{S n *}, \ldots, Y_{k}^{S n *}\right\}, \\
Y^{*}=\left\{Y_{1}^{1 *}, \ldots, Y_{k}^{1 *}, \ldots, Y_{1}^{n *}, \ldots, Y_{k}^{n *}\right\}, \\
Y^{I *}=\left\{Y_{1}^{I 1 *}, \ldots, Y_{k}^{I 1 *}, \ldots, Y_{1}^{I n *}, \ldots, Y_{k}^{I n *}\right\}, \\
Y^{I S *}=\left\{Y_{1}^{I S 1 *}, \ldots, Y_{k}^{I S 1 *}, \ldots, Y_{1}^{I S n *}, \ldots, Y_{k}^{I S n *}\right\}, \\
Y^{I M *}=\left\{Y_{1}^{I M 1 *}, \ldots, Y_{k}^{I M 1 *}, \ldots, Y_{1}^{I M n *}, \ldots, Y_{k}^{I M n *}\right\},
\end{gathered}
$$

and

$$
\begin{aligned}
Y^{I S p *}= & \left\{\operatorname{spr} Y_{1}^{1}+\left|G_{1}^{1 *}\right|, \ldots, \operatorname{spr} Y_{k}^{1}+\left|G_{k}^{1 *}\right|, \ldots, \operatorname{spr} Y_{1}^{n}+\left|G_{1}^{n *}\right|, \ldots, \operatorname{spr} Y_{k}^{n}\right. \\
& \left.+\left|G_{k}^{n *}\right|\right\} .
\end{aligned}
$$

\section{Provided Estimators}

The motivation of this article is to propose new estimators for the parameters of the model (1) that to be superior to the old known estimators of the parameters in Blanco-Fernández et al. (2011). Based on the new structure of MCR in Section 3, Section 4.2 proposes the new estimators of the 
parameters, and also Section 4.1 re-introduces the old known estimators of the parameters.

\subsection{The Old Known Estimators}

Blanco-Fernández et al. (2011) solved the LS estimation of the model (1) over a suitable feasible set which assures the coherence of the solutions has been proposed and analytically solved.

By considering Section 3, $\operatorname{spr} X_{i}^{b}, X_{i}^{S b *}, X_{i}^{M b *}, X_{i}^{b *}, Y_{i}^{M b *}, \operatorname{spr} Y_{i}^{b}$, $Y_{i}^{S b *}$, and $Y_{i}^{b *}$ are the $i$ th element of the sample sets $X^{S p * b}, X^{S * b}, X^{M * b}$, $X^{* b}, Y^{M * b}, Y^{S p * b}, Y^{S * b}$, and $Y^{* b}$ given in (7)-(14), respectively, $i=1, \ldots, k$ and $b=1, \ldots, n$. Also, $X_{j}^{S p *}, X_{j}^{S *}, X_{j}^{M *}, X_{j}^{*}, Y_{j}^{M *}, Y_{j}^{S p *}, Y_{j}^{S *}$, and $Y_{j}^{*}$, $j=1, \ldots, n k$, are defined as the $j$ th element of the sample sets $X^{S p *}, X^{S *}$, $X^{M *}, X^{*}, Y^{M *}, Y^{S p *}, Y^{S *}$, and $Y^{*}$ given in (23)-(30), respectively.

This section re-introduces the old known estimators of the parameters of the model (1) presented in Blanco-Fernández et al. (2011) according to the random sample $\left\{\left(X_{j}^{*}, Y_{j}^{*}\right)\right\}_{j=1}^{n k}$ from $(X, Y)$ as follows:

$$
\begin{gathered}
\widehat{\alpha}=\frac{\widehat{\sigma}\left(X^{M}, Y\right)}{\widehat{\sigma}^{2}\left(X^{M}\right)}=\frac{\operatorname{Cov}\left(X^{M *}, Y^{*}\right)}{\operatorname{Var}\left(X^{M *}\right)} \\
\widehat{\beta}=\min \left\{\widehat{u}_{0}, \max \left\{0, \frac{\widehat{\sigma}\left(X^{S}, Y\right)}{\widehat{\sigma}^{2}\left(X^{S}\right)}\right\}\right\}=\min \left\{\widehat{u}_{0}, \max \left\{0, \frac{\operatorname{Cov}\left(X^{S *}, Y^{*}\right)}{\operatorname{Var}\left(X^{S *}\right)}\right\}\right\} \\
\widehat{\gamma}=\overline{Y^{M *}}-\widehat{\alpha} \overline{X^{M *}},
\end{gathered}
$$

and

$$
\widehat{\delta}=\overline{Y^{S p *}}-\widehat{\beta} \overline{X^{S p *}},
$$

where $\widehat{u}_{0}=\min \left\{\frac{Y_{j}^{S p *}}{X_{j}^{S p *}}: X_{j}^{S p *} \neq 0\right\}$ and $\overline{Y^{M *}}=\frac{1}{n k} \sum_{j=1}^{n k} Y_{j}^{M *}$ (analogously $\overline{X^{M *}}, \overline{Y^{S p *}}$, and $\overline{X^{S p *}}$. The estimators are provided under the condition $n k \rightarrow \infty$. Also, when $n, k$ tend to infinity, the old known estimators (35)(38), respectively, can be shown as

$$
\widehat{\alpha}=\frac{1}{n} \sum_{b=1}^{n} \widehat{\alpha^{b}}=\frac{1}{n} \sum_{b=1}^{n}\left(\frac{\operatorname{Cov}\left(X^{M * b}, Y^{* b}\right)}{\operatorname{Var}\left(X^{M * b}\right)}\right),
$$




$$
\begin{gathered}
\widehat{\beta}=\frac{1}{n} \sum_{b=1}^{n} \widehat{\beta^{b}}=\frac{1}{n} \sum_{b=1}^{n}\left(\operatorname { m i n } \left\{\min \left\{\frac{\operatorname{spr} Y_{i}^{b}}{\operatorname{spr} X_{i}^{b}}: \operatorname{spr} X_{i}^{b} \neq 0\right\}\right.\right. \\
\left.\left.\max \left\{0, \frac{\operatorname{Cov}\left(X^{S * b}, Y^{* b}\right)}{\operatorname{Var}\left(X^{S * b}\right)}\right\}\right\}\right) \\
\widehat{\gamma}=\frac{1}{n} \sum_{b=1}^{n} \widehat{\gamma^{b}}=\frac{1}{n} \sum_{b=1}^{n}\left(\overline{Y^{M * b}}-\widehat{\alpha^{b}} \overline{X^{M * b}}\right),
\end{gathered}
$$

and

$$
\widehat{\delta}=\frac{1}{n} \sum_{b=1}^{n} \widehat{\delta^{b}}=\frac{1}{n} \sum_{b=1}^{n}\left(\overline{Y^{S p * b}}-\widehat{\beta^{b}} \overline{X^{S p * b}}\right),
$$

where $\overline{Y^{M * b}}=\frac{1}{k} \sum_{i=1}^{k} Y_{i}^{M b *}$ (analogously $\overline{X^{M * b}}, \overline{Y^{S p * b}}$, and $\overline{X^{S p * b}}$ ).

\subsection{New Estimators}

Another novelty and innovation of this paper is to introduce the new estimators for the parameters of the model (1). According to Section 3, $\operatorname{spr} X_{i}^{b}$, $X_{i}^{S b *}, X_{i}^{M b *}, X_{i}^{b *}, Y_{i}^{I b *}, Y_{i}^{I S b *}, Y_{i}^{I M b *}$, and $\operatorname{spr} Y_{i}^{b}+\left|G_{i}^{* b}\right|$ are the $i$ th element of the sample sets $X^{S p * b}, X^{S * b}, X^{M * b}, X^{* b}, Y^{I * b}, Y^{I S * b}, Y^{I M * b}$, and $Y^{I S p * b}$ given in (7)-(10) and (15)-(18), respectively, $i=1, \ldots, k$ and $b=1, \ldots, n$. Also, $X_{j}^{S p *}, X_{j}^{S *}, X_{j}^{M *}, X_{j}^{*}, Y_{j}^{I *}, Y_{j}^{I S *}, Y_{j}^{I M *}$, and $Y_{j}^{I S p *}, j=1, \ldots, n k$, are defined as the $j$ th element of the sample sets $X^{S p *}, X^{S *}, X^{M *}, X^{*}, Y^{I *}$, $Y^{I S *}, Y^{I M *}$, and $Y^{I S p *}$ given in (23)-(26) and (31)-(34), respectively.

The new estimators for the parameters of the model (1) are proposed on the basis of the random sample $\left\{\left(X_{j}^{*}, Y_{j}^{I *}\right)\right\}_{j=1}^{n k}$ from $\left(X, Y^{I}\right)$ as follows:

$$
\begin{gathered}
\widehat{\alpha}=\frac{\widehat{\sigma}\left(X^{M}, Y^{I}\right)}{\widehat{\sigma}^{2}\left(X^{M}\right)}=\frac{\operatorname{Cov}\left(X^{M *}, Y^{I *}\right)}{\operatorname{Var}\left(X^{M *}\right)}, \\
\widehat{\beta}=\min \left\{\min \left\{\frac{Y_{j}^{I S p *}}{X_{j}^{S p *}}: X_{j}^{S p *} \neq 0\right\}, \max \left\{0, \frac{\widehat{\sigma}\left(X^{S}, Y^{I}\right)}{\widehat{\sigma}^{2}\left(X^{S}\right)}\right\}\right\} \\
=\min \left\{\min \left\{\frac{Y_{j}^{I S p *}}{X_{j}^{S p *}}: X_{j}^{S p *} \neq 0\right\}, \max \left\{0, \frac{\operatorname{Cov}\left(X^{S *}, Y^{I *}\right)}{\operatorname{Var}\left(X^{S *}\right)}\right\}\right\},
\end{gathered}
$$




$$
\widehat{\gamma}=\overline{Y^{I M *}}-\widehat{\alpha} \overline{X^{M *}}
$$

and

$$
\widehat{\delta}=\overline{Y^{I S p *}}-\widehat{\beta} \overline{X^{S p *}},
$$

where $\overline{Y^{I M *}}=\frac{1}{n k} \sum_{j=1}^{n k} Y_{j}^{I M *}$ (analogously $\overline{Y^{I S p *}}, \overline{X^{M *}}$, and $\overline{X^{S p *}}$ ). The new estimators are provided under the conditions $n k \rightarrow \infty$ and $Y^{I}-Y \stackrel{\mathcal{L}}{\longrightarrow} \underline{0}$ (that is $E\left(Y^{I}-Y\right) \rightarrow 0$ and $\sigma^{2}\left(Y^{I}-Y\right) \rightarrow 0$ ). Also, when $n, k \rightarrow \infty$ and $Y^{I}-Y \stackrel{\mathcal{L}}{\longrightarrow} \underline{0}$, we can write the new estimators (39)-(42), respectively, as follows:

$$
\begin{aligned}
& \widehat{\alpha}=\frac{1}{n} \sum_{b=1}^{n} \widehat{\alpha^{b}}=\frac{1}{n} \sum_{b=1}^{n}\left(\frac{\operatorname{Cov}\left(X^{M * b}, Y^{I * b}\right)}{\operatorname{Var}\left(X^{M * b}\right)}\right), \\
& \widehat{\beta}=\frac{1}{n} \sum_{b=1}^{n} \widehat{\beta}^{b}=\frac{1}{n} \sum_{b=1}^{n}\left(\operatorname { m i n } \left\{\min \left\{\frac{\operatorname{spr} Y_{i}^{b}+\left|G_{i}^{b *}\right|}{\operatorname{spr} X_{i}^{b}}: \operatorname{spr} X_{i}^{b} \neq 0\right\}\right.\right. \text {, } \\
& \left.\left.\max \left\{0, \frac{\operatorname{Cov}\left(X^{S * b}, Y^{I * b}\right)}{\operatorname{Var}\left(X^{S * b}\right)}\right\}\right\}\right) \text {, } \\
& \widehat{\gamma}=\frac{1}{n} \sum_{b=1}^{n} \widehat{\gamma^{b}}=\frac{1}{n} \sum_{b=1}^{n}\left(\overline{Y^{I M * b}}-\widehat{\alpha^{b}} \overline{X^{M * b}}\right),
\end{aligned}
$$

and

$$
\widehat{\delta}=\frac{1}{n} \sum_{b=1}^{n} \widehat{\delta^{b}}=\frac{1}{n} \sum_{b=1}^{n}\left(\overline{Y^{I S p * b}}-\widehat{\beta^{b}} \overline{X^{S p * b}}\right),
$$

where $\overline{Y^{I M * b}}=\frac{1}{k} \sum_{i=1}^{k} Y_{i}^{I M b *}$ (analogously $\overline{Y^{I S p * b}}, \overline{X^{M * b}}$, and $\overline{X^{S p * b}}$ ).

\section{Optimal Properties of the Estimators}

We utilize the new structure defined in Section 3, which accounts the internal variations of the intervals, to fit the model (1) on interval-valued data.

The strong consistency, the asymptotic unbiasedness, the asymptotic distribution, the asymptotic variance, and the asymptotic mean squared error (MSE) of each of the estimators (35)-(42) are studied and demonstrated under the conditions $n k \rightarrow \infty$ and $Y-Y^{I} \stackrel{\mathcal{L}}{\longrightarrow} \underline{0}$ in this section. Hence, this section investigates the theoretical performance of the estimators. In this paper, we express the old known and new estimators of the parameters of 
the model (1) briefly as the old known estimators and the new estimators, respectively.

\subsection{Optimal Properties of the Old Known Estimators}

In this section, we investigate the optimal properties (the asymptotic unbiasedness and the strong consistency) of each of the old known estimators (see the estimators (35)-(38)) when $n k \rightarrow \infty$. Also, the asymptotic distributions, the asymptotic variances, and the asymptotic MSEs of some of the old estimators are proposed.

Theorem 1. Let $X, Y$ be random intervals verifying a model (1), such that $\sigma(Y, X)<\infty$, and $0<\sigma^{2}(Y), \sigma^{2}(X)<\infty$. The old known estimators are strongly consistent with respect to the parameters of the model (1).

Proof. From $\sigma(Y, X)<\infty$ and $0<\sigma^{2}(Y), \sigma^{2}(X)<\infty$, we can conclude, respectively, $\sigma\left(X^{M}, Y^{M}\right), \sigma\left(X^{S}, Y^{S}\right)<\infty$ and $0<\sigma^{2}\left(Y^{M}\right), \sigma^{2}\left(Y^{S}\right)$, $\sigma^{2}\left(X^{M}\right), \sigma^{2}\left(X^{S}\right)<\infty$ (see Section 2.1).

The Strong Law of Large Numbers guarantees that the terms $\widehat{\sigma}\left(X^{M}, Y\right)=$ $\widehat{\sigma}\left(X^{M}, Y^{M}\right)$ (because the variable $Y$ is $Y^{M}+Y^{S}$, and the variable $Y^{S}$ is independent from the variable $\left.X^{M}\right)$ and $\widehat{\sigma}^{2}\left(X^{M}\right)$ converge almost surely to $\sigma\left(X^{M}, Y\right)=\sigma\left(X^{M}, Y^{M}\right)$ and $\sigma^{2}\left(X^{M}\right)$, respectively. Thus, based on the Slutzky Theorem, we conclude that the old known estimator $\widehat{\alpha}=\frac{\widehat{\sigma}\left(X^{M}, Y\right)}{\widehat{\sigma}^{2}\left(X^{M}\right)}$ in $(35)$ converges a.s. $-[\mathrm{P}]$ to the parameter $\alpha=\frac{\sigma\left(X^{M}, Y\right)}{\sigma^{2}\left(X^{M}\right)}$ (this means $\widehat{\alpha} \stackrel{n k \rightarrow \infty}{\longrightarrow} \alpha$ a.s. $-[\mathrm{P}])$.

The strong consistency of the estimator $\widehat{\gamma}=\overline{Y^{M *}}-\widehat{\alpha} \overline{X^{M *}}$ in (37) with respect to the parameter $\gamma=E\left(Y^{M}\right)-\alpha E\left(X^{M}\right)$ follows from the convergence of $\widehat{\alpha}$ in (35), together with the strong law of large numbers for the sample mean of an interval random set (see Artstein and Vitale , 1975). Hence, we conclude $\widehat{\gamma} \stackrel{n k \rightarrow \infty}{\longrightarrow} \gamma$ a.s. $-[\mathrm{P}]$.

For $\widehat{\beta}$ given in (36), we are going to prove $\widehat{\beta} \stackrel{n k \rightarrow \infty}{\longrightarrow} \beta$ a.s. $-[\mathrm{P}]$. First, we will demonstrate that $T_{n k}=\max \left\{0, \frac{\widehat{\sigma}\left(X^{S}, Y\right)}{\widehat{\sigma}^{2}\left(X^{S}\right)}\right\}=\max \left\{0, \widehat{\beta}_{1}\right\} \stackrel{n k \rightarrow \infty}{\longrightarrow} \beta=$ $\frac{\sigma\left(X^{S}, Y\right)}{\sigma^{2}\left(X^{S}\right)}$ a.s. $-[\mathrm{P}]$. The expressions $\widehat{\sigma}\left(X^{S}, Y\right)=\widehat{\sigma}\left(X^{S}, Y^{S}\right)$ (because the variable $Y$ is $Y^{S}+Y^{M}$, and the variable $Y^{M}$ is independent from the variable $\left.X^{S}\right)$ and $\widehat{\sigma}^{2}\left(X^{S}\right)$ converge almost surely to $\sigma\left(X^{S}, Y\right)=\sigma\left(X^{S}, Y^{S}\right)$ 
and $\sigma^{2}\left(X^{S}\right)$, respectively, using the Strong Law of Large Numbers. Thus, based on the Slutzky Theorem, we conclude $\widehat{\beta}_{1} \stackrel{n k \rightarrow \infty}{\longrightarrow} \beta$ a.s. $-[\mathrm{P}]$. By considering the real continuous mapping $\max \{0, \cdot\}$, the expression $T_{n k}=$ $\max \left\{0, \widehat{\beta}_{1}\right\} \stackrel{n k \rightarrow \infty}{\longrightarrow} \max \{0, \beta\}$ a.s. $-[\mathrm{P}]$ can be obtained. We have $\max \{0, \beta\}$ $=\beta$ in $T_{n k}=\max \left\{0, \widehat{\beta}_{1}\right\} \stackrel{n k \rightarrow \infty}{\longrightarrow} \max \{0, \beta\}$ a.s. $-[\mathrm{P}]$ when $0<\sigma^{2}\left(X^{S}\right)<\infty$, and $\beta \geqslant 0$. Hence, we conclude $T_{n k} \stackrel{n k \rightarrow \infty}{\longrightarrow} \beta$ a.s. $-[\mathrm{P}]$.

We introduce $u_{0}=\min \left\{\frac{\operatorname{spr} Y_{o}}{\operatorname{spr} X_{o}}: \operatorname{spr} X_{o} \neq 0\right\}, o=1, \ldots, N$, where $N$ is the number of members of the population. Hence, the expression $P\left(\lim _{n k \rightarrow \infty}\right.$ $\left.\widehat{u}_{0}(n k)=u_{0}\right)=P\left(\lim _{n k \rightarrow N} \widehat{u}_{0}(n k)=u_{0}\right)=1$ is easily proven. The result $\min \left\{u_{0}, T_{n k}\right\} \leqslant \min \left\{\widehat{u}_{0}, T_{n k}\right\}=\widehat{\beta}$ can be obtained on the basis of $\widehat{\beta} \leqslant$ $T_{n k}$, and also $u_{0} \leqslant \widehat{u}_{0}$, where $\widehat{u}_{0}$ is considered as the sample of $u_{0}$. Thus, we have $\min \left\{u_{0}, T_{n k}\right\} \leqslant \widehat{\beta} \leqslant T_{n k}$. By considering $\beta, \operatorname{spr} X, \operatorname{spr} \varepsilon \geqslant 0$ in the model $\operatorname{spr} Y=\beta \operatorname{spr} X+\operatorname{spr} \varepsilon$, the conclusion $\min \left\{u_{0}, \beta\right\}=\beta$ can be expressed. The convergence $\min \left\{u_{0}, T_{n k}\right\} \stackrel{n k \rightarrow \infty}{\longrightarrow} \min \left\{u_{0}, \beta\right\}=\beta$ a.s. $-[\mathrm{P}]$ can be concluded using the real continuous mapping $\min \left\{u_{0}, \cdot\right\}$. So, the convergence $\widehat{\beta} \stackrel{n k \rightarrow \infty}{\longrightarrow} \beta$ a.s. $-[\mathrm{P}]$ is proven using the convergence $\min \left\{u_{0}, T_{n k}\right\}$ $\stackrel{n k \rightarrow \infty}{\longrightarrow} \min \left\{u_{0}, \beta\right\}=\beta$ a.s. $-[\mathrm{P}]$, the conclusion $T_{n k} \stackrel{n k \rightarrow \infty}{\longrightarrow} \beta$ a.s. $-[\mathrm{P}]$, and the sandwich rule in $\min \left\{u_{0}, T_{n k}\right\} \leqslant \widehat{\beta} \leqslant T_{n k}$.

By considering $\widehat{\beta} \stackrel{n k \rightarrow \infty}{\longrightarrow} \beta$ a.s. $-[\mathrm{P}]$ and the strong law of large numbers for the sample mean of an interval random set, the strong consistency of the estimator $\widehat{\delta}=\overline{Y^{S p *}}-\widehat{\beta} \overline{X^{S p *}}$ in (38) with respect to the parameter $\delta=$ $E(\operatorname{spr} Y)-\beta E(\operatorname{spr} X)$ is proven. Hence, we can express $\widehat{\delta} \stackrel{n k \rightarrow \infty}{\longrightarrow} \delta$ a.s. $-[\mathrm{P}]$.

Theorem 2. Let $X, Y$ be random intervals verifying a model (1), such that $0<\sigma^{2}(Y), \sigma^{2}(X)<\infty$, and $\sigma\left(X^{M}, Y^{M}\right), \sigma\left(X^{S}, Y^{S}\right)<\infty$. The old known estimators are asymptotically unbiased.

Proof. Section 2.1 shows that if we have $0<\sigma^{2}(Y), \sigma^{2}(X)<\infty$ and $\sigma\left(X^{M}, Y^{M}\right), \sigma\left(X^{S}, Y^{S}\right)<\infty$, then we can express $0<\sigma^{2}\left(Y^{M}\right), \sigma^{2}\left(Y^{S}\right)$, $\sigma^{2}\left(X^{M}\right), \sigma^{2}\left(X^{S}\right)<\infty$ and $\sigma(X, Y)<\infty$, respectively. Considering Section 2.1 and the definition of $Y=Y^{S}+Y^{M}$ in (2) (we know $Y^{S}=\beta X^{S}+\varepsilon^{S}$ and $\left.Y^{M}=\alpha X^{M}+\gamma[1 \pm 0]+\varepsilon^{M}\right)$, we conclude $\sigma\left(X^{S}, \varepsilon^{S}\right), \sigma\left(X^{M}, \varepsilon^{M}\right)<\infty$ from $\sigma\left(X^{S}, Y^{S}\right), \sigma\left(X^{M}, Y^{M}\right)<\infty$ and $0<\sigma^{2}\left(X^{S}\right), \sigma^{2}\left(X^{M}\right)<\infty$. As a result, we will express $\sigma(X, \varepsilon)=\sigma\left(X^{S}, \varepsilon^{S}\right)+\sigma\left(X^{M}, \varepsilon^{M}\right)<\infty$. 
The old known estimator $\widehat{\alpha}=\frac{\widehat{\sigma}\left(X^{M}, Y\right)}{\widehat{\sigma}^{2}\left(X^{M}\right)}=\frac{\widehat{\sigma}\left(X^{M}, Y^{M}+Y^{S}\right)}{\widehat{\sigma}^{2}\left(X^{M}\right)}$ in (35) can be expressed as $\widehat{\alpha}=\frac{\widehat{\sigma}\left(X^{M}, Y^{M}\right)}{\widehat{\sigma}^{2}\left(X^{M}\right)}$, because the variables $X^{M}$ and $Y^{S}$ are independent from each other. The estimator $\frac{\widehat{\sigma}\left(X^{M}, Y^{M}\right)}{\widehat{\sigma}^{2}\left(X^{M}\right)}$ is the LS estimator of the parameter $\alpha$ of the linear model $Y^{M}=\alpha X^{M}+\gamma[1 \pm 0]+\varepsilon^{M}$. Taking into account that $Y_{j}^{M *}=\alpha X_{j}^{M *}+\gamma+\varepsilon_{j}^{M *}$ for all $j=1, \ldots, n k$ (see the $j$ th element of the sets $\varepsilon^{M *}, X^{M *}$, and $Y^{M *}$, respectively, given in (19), (25), and (27)), the expression $\widehat{\alpha}-\alpha=\frac{\widehat{\sigma}\left(X^{M}, \varepsilon^{M}\right)}{\widehat{\sigma}^{2}\left(X^{M}\right)}$ is concluded. Based on the strong consistency of $\widehat{\sigma}\left(X^{M}, \varepsilon^{M}\right)$ and $\widehat{\sigma}^{2}\left(X^{M}\right)$ with respect to $\sigma\left(X^{M}, \varepsilon^{M}\right)$ and $\sigma^{2}\left(X^{M}\right)$, respectively, we conclude $\frac{\widehat{\sigma}\left(X^{M}, \varepsilon^{M}\right)}{\widehat{\sigma}^{2}\left(X^{M}\right)} \stackrel{n k \rightarrow \infty}{\longrightarrow} \frac{\sigma\left(X^{M}, \varepsilon^{M}\right)}{\sigma^{2}\left(X^{M}\right)}$ a.s. - $[\mathrm{P}]$. Hence, when $n k \rightarrow \infty, E(\widehat{\alpha})-\alpha$ and $\frac{\sigma\left(X^{M}, \varepsilon^{M}\right)}{\sigma^{2}\left(X^{M}\right)}$ are (asymptotically) equivalent; in symbols: $\frac{E(\widehat{\alpha})-\alpha}{\left(\frac{\sigma\left(X^{M}, \varepsilon^{M}\right)}{\sigma^{2}\left(X^{M}\right)}\right)} \rightarrow 1$ or $E(\widehat{\alpha})-\alpha \approx \frac{\sigma\left(X^{M}, \varepsilon^{M}\right)}{\sigma^{2}\left(X^{M}\right)}$. Based on Theorem 1, we have $\widehat{\alpha} \stackrel{n k \rightarrow \infty}{\longrightarrow} \alpha$ a.s. $-[\mathrm{P}]$, and hence $E(\widehat{\alpha})-\alpha \approx 0$. So, the old known estimator $\widehat{\alpha}$ in $(35)$ is asymptotically unbiased. Hence, we can conclude $E(\widehat{\alpha})-\alpha \approx \frac{\sigma\left(X^{M}, \varepsilon^{M}\right)}{\sigma^{2}\left(X^{M}\right)} \approx 0$.

Since the variables $X^{S}$ and $Y^{M}$ are independent from each other, the expression $\frac{\widehat{\sigma}\left(X^{S}, Y\right)}{\widehat{\sigma}^{2}\left(X^{S}\right)}=\frac{\widehat{\sigma}\left(X^{S}, Y^{M}+Y^{S}\right)}{\widehat{\sigma}^{2}\left(X^{S}\right)}$ in $(36)$ can be written as $\frac{\widehat{\sigma}\left(X^{S}, Y^{S}\right)}{\widehat{\sigma}^{2}\left(X^{S}\right)}$. The estimator $\frac{\widehat{\sigma}\left(X^{S}, Y^{S}\right)}{\widehat{\sigma}^{2}\left(X^{S}\right)}$ is an estimator for the parameter $\beta$ of the model $Y^{S}=\beta X^{S}+\varepsilon^{S}$. Taking into account that $Y_{j}^{S *}=\beta X_{j}^{S *}+\varepsilon_{j}^{S *}$ for all $j=1, \ldots, n k$ (see the $j$ th element of the sets $\varepsilon^{S *}, X^{S *}$, and $Y^{S *}$, respectively, given in (21), (24), and (29)), the expression $\frac{\widehat{\sigma}\left(X^{S}, Y^{S}\right)}{\widehat{\sigma}^{2}\left(X^{S}\right)}-\beta=\frac{\widehat{\sigma}\left(X^{S}, \varepsilon^{S}\right)}{\widehat{\sigma}^{2}\left(X^{S}\right)}$ can be concluded. The Strong Law of Large Numbers guarantees that the terms $\widehat{\sigma}\left(X^{S}, \varepsilon^{S}\right)$ and $\widehat{\sigma}^{2}\left(X^{S}\right)$ converge almost surely to $\sigma\left(X^{S}, \varepsilon^{S}\right)$ and $\sigma^{2}\left(X^{S}\right)$, respectively. Thus, based on the Slutzky Theorem, we conclude $\frac{\widehat{\sigma}\left(X^{S}, \varepsilon^{S}\right)}{\widehat{\sigma}^{2}\left(X^{S}\right)} \stackrel{P}{\rightarrow} \frac{\sigma\left(X^{S}, \varepsilon^{S}\right)}{\sigma^{2}\left(X^{S}\right)}$. Hence, when $n k \rightarrow \infty$, we can write $E\left(\frac{\widehat{\sigma}\left(X^{S}, Y^{S}\right)}{\widehat{\sigma}^{2}\left(X^{S}\right)}\right)-$ $\beta=E\left(\frac{\widehat{\sigma}\left(X^{S}, \varepsilon^{S}\right)}{\widehat{\sigma}^{2}\left(X^{S}\right)}\right) \approx \frac{\sigma\left(X^{S}, \varepsilon^{S}\right)}{\sigma^{2}\left(X^{S}\right)}$. By considering the proof of Theorem 1, we have $\frac{\widehat{\sigma}\left(X^{S}, Y\right)}{\widehat{\sigma}^{2}\left(X^{S}\right)} \stackrel{n k \rightarrow \infty}{\longrightarrow} \beta=\frac{\sigma\left(X^{S}, Y\right)}{\sigma^{2}\left(X^{S}\right)}$ a.s. $-[\mathrm{P}]$ and $\widehat{\beta} \stackrel{n k \rightarrow \infty}{\longrightarrow} \beta$ a.s. $-[\mathrm{P}]$. Hence, we conclude $E\left(\frac{\widehat{\sigma}\left(X^{S}, Y^{S}\right)}{\widehat{\sigma}^{2}\left(X^{S}\right)}\right)-\beta \approx 0$ and $E(\widehat{\beta})-\beta \approx 0$. So, the estimator 
$\widehat{\beta}$ in (36) is asymptotically unbiased. By considering $E\left(\frac{\widehat{\sigma}\left(X^{S}, Y^{S}\right)}{\widehat{\sigma}^{2}\left(X^{S}\right)}\right)-\beta \approx$ $\frac{\sigma\left(X^{S}, \varepsilon^{S}\right)}{\sigma^{2}\left(X^{S}\right)}, E\left(\frac{\widehat{\sigma}\left(X^{S}, Y^{S}\right)}{\widehat{\sigma}^{2}\left(X^{S}\right)}\right)-\beta \approx 0$, and $E(\widehat{\beta})-\beta \approx 0$, we can conclude $E(\widehat{\beta})-\beta \approx \frac{\sigma\left(X^{S}, \varepsilon^{S}\right)}{\sigma^{2}\left(X^{S}\right)} \approx 0$.

Based on the result $\widehat{\alpha}=\alpha+\frac{\widehat{\sigma}\left(X^{M}, \varepsilon^{M}\right)}{\widehat{\sigma}^{2}\left(X^{M}\right)}$ provided in the proof of the asymptotic unbiasedness of the estimator $(35)$, we write $E(\widehat{\gamma})=E\left(\overline{Y^{M *}}-\widehat{\alpha} \overline{X^{M *}}\right)=$ $\frac{1}{n k} \sum_{j=1}^{n k} E\left(Y_{j}^{M *}\right)-E\left(\left(\alpha+\frac{\widehat{\sigma}\left(X^{M}, \varepsilon^{M}\right)}{\widehat{\sigma}^{2}\left(X^{M}\right)}\right) \overline{X^{M *}}\right)=\alpha X^{M}+\gamma-\alpha X^{M}-$ $E\left(\frac{\widehat{\sigma}\left(X^{M}, \varepsilon^{M}\right)}{\widehat{\sigma}^{2}\left(X^{M}\right)} \overline{X^{M *}}\right)$. Hence, we conclude $E(\widehat{\gamma})-\gamma=-E\left(\frac{\widehat{\sigma}\left(X^{M}, \varepsilon^{M}\right)}{\widehat{\sigma}^{2}\left(X^{M}\right)} \overline{X^{M *}}\right)$. Also, based on Theorem 1, we express $\widehat{\gamma} \stackrel{n k \rightarrow \infty}{\longrightarrow} \gamma$ a.s. $-[\mathrm{P}]$, and hence $E(\widehat{\gamma})-\gamma \approx 0$ (this means that the estimator $\widehat{\gamma}$ in (37) is asymptotically unbiased). Hence, we can write $E(\widehat{\gamma})-\gamma=-E\left(\frac{\widehat{\sigma}\left(X^{M}, \varepsilon^{M}\right)}{\widehat{\sigma}^{2}\left(X^{M}\right)} \overline{X^{M *}}\right) \approx 0$.

By considering the asymptotic unbiasedness of $\widehat{\beta}$ in $(36), E(\widehat{\delta})=E\left(\overline{Y^{S p *}}\right.$ $\left.-\widehat{\beta} \overline{X^{S p *}}\right) \approx \frac{1}{n k} \sum_{j=1}^{n k} E\left(Y_{j}^{S p *}\right)-E\left(\left(\beta+\frac{\widehat{\sigma}\left(X^{S}, \varepsilon^{S}\right)}{\widehat{\sigma}^{2}\left(X^{S}\right)}\right) \overline{X^{S p *}}\right)=\beta \operatorname{spr} X+$ $\delta-\beta \operatorname{spr} X-E\left(\frac{\widehat{\sigma}\left(X^{S}, \varepsilon^{S}\right)}{\widehat{\sigma}^{2}\left(X^{S}\right)} \overline{X^{S p *}}\right)$ can be obtained, so $E(\widehat{\delta})-\delta \approx-E\left(\frac{\widehat{\sigma}\left(X^{S}, \varepsilon^{S}\right)}{\widehat{\sigma}^{2}\left(X^{S}\right)}\right.$ $\overline{X^{S p *}}$ ) can be concluded. Based on Theorem 1, the asymptotic unbiasedness of the estimator $\widehat{\delta}$ in (38) is concluded. Hence, $E(\widehat{\delta})-\delta \approx-E\left(\frac{\widehat{\sigma}\left(X^{S}, \varepsilon^{S}\right)}{\widehat{\sigma}^{2}\left(X^{S}\right)} \overline{X^{S p *}}\right)$ $\approx 0$ can be expressed.

The estimators provided by Blanco-Fernández et al. (2011) are the same the old known estimators. The results obtained about the strong consistency and the asymptotic unbiasedness of the old known estimators (see Theorems 1 and 2, respectively) confirm the results obtained about the corresponding properties of the estimators in Blanco-Fernández et al. (2011). In the mentioned paper, in contrary to this article, there is no study on the asymptotic distributions, the asymptotic variances, and the asymptotic MSEs of the estimators (the contributions of this research). The asymptotic distribution, the asymptotic variance, and the asymptotic MSE of each of the old known estimators (35) and (36) are studied in the rest of this section. 
Theorem 3. Assume that $X, Y$ be random intervals verifying a model (1). If $0<\sigma^{2}\left(Y^{M}\right), \sigma^{2}\left(Y^{S}\right), \sigma^{2}\left(X^{M}\right), \sigma^{2}\left(X^{S}\right)<\infty, \sigma\left(X^{M}, Y^{M}\right), \sigma\left(X^{S}, Y^{S}\right)$ $<\infty, \sigma^{2}\left(X^{M}\right), \sigma^{2}\left(X^{S}\right) \nrightarrow 0$, and as the results of the assumptions, $0<$ $\frac{\sigma^{2}\left(\varepsilon^{M}\right)}{\sigma^{2}\left(X^{M}\right)}, \frac{\sigma^{2}\left(\varepsilon^{S}\right)}{\sigma^{2}\left(X^{S}\right)}<\infty$, then

$$
\sqrt{n k}(\widehat{\alpha}-\alpha) \stackrel{\mathcal{L}}{\longrightarrow} N\left(0, \frac{\sigma^{2}\left(\varepsilon^{M}\right)}{\sigma^{2}\left(X^{M}\right)}\right),
$$

and

$$
\sqrt{n k}\left(\widehat{\beta}_{1}-\beta\right) \stackrel{\mathcal{L}}{\longrightarrow} N\left(0, \frac{\sigma^{2}\left(\varepsilon^{S}\right)}{\sigma^{2}\left(X^{S}\right)}\right),
$$

where $\widehat{\alpha}$ and $\widehat{\beta}_{1}=\frac{\widehat{\sigma}\left(Y, X^{S}\right)}{\widehat{\sigma}^{2}\left(X^{S}\right)}$, respectively, are the estimators provided in (35) and (36).

A unique expression dose not exist for the distribution of the old known estimator $\widehat{\beta}$ in (36) because $\widehat{\beta}$ depends on min and max operators. Hence, a valid asymptotic distribution is proposed by using the partial information $\widehat{\beta}_{1}=\frac{\widehat{\sigma}\left(Y, X^{S}\right)}{\widehat{\sigma}^{2}\left(X^{S}\right)}$. The asymptotic distributions of the estimators $\widehat{\alpha}$ and $\widehat{\beta}_{1}$ are introduced, respectively, as the distributions (43) and (44).

Proof. Section 2.1 shows that if we have $0<\sigma^{2}\left(Y^{M}\right), \sigma^{2}\left(Y^{S}\right), \sigma^{2}\left(X^{M}\right)$, $\sigma^{2}\left(X^{S}\right)<\infty$ and $\sigma\left(X^{M}, Y^{M}\right), \sigma\left(X^{S}, Y^{S}\right)<\infty$, then we can conclude $0<\sigma^{2}(Y), \sigma^{2}(X)<\infty$ and $\sigma(X, Y)<\infty$, respectively. Hence, considering Section 2.1 and the definition of the interval variable $Y=Y^{M}+Y^{S}$ in (2) (with $Y^{M}=\alpha X^{M}+\gamma[1 \pm 0]+\varepsilon^{M}$ and $Y^{S}=\beta X^{S}+\varepsilon^{S}$ ), we conclude $0<\sigma^{2}(\varepsilon), \sigma^{2}\left(\varepsilon^{M}\right), \sigma^{2}\left(\varepsilon^{S}\right)<\infty$ and $\sigma(X, \varepsilon), \sigma\left(X^{M}, \varepsilon^{M}\right), \sigma\left(X^{S}, \varepsilon^{S}\right)<\infty$ from the assumptions of the theorem and the results of the assumptions. As results, if we have $\sigma^{2}\left(X^{M}\right), \sigma^{2}\left(X^{S}\right) \nrightarrow 0$, then $0<\frac{\sigma^{2}\left(\varepsilon^{M}\right)}{\sigma^{2}\left(X^{M}\right)}, \frac{\sigma^{2}\left(\varepsilon^{S}\right)}{\sigma^{2}\left(X^{S}\right)}<\infty$ will be confirmed.

In the proof of Theorem 2, we showed that $\widehat{\alpha}=\frac{\widehat{\sigma}\left(Y, X^{M}\right)}{\widehat{\sigma}^{2}\left(X^{M}\right)}$ in (35) can be written as $\widehat{\alpha}=\frac{\widehat{\sigma}\left(Y^{M}, X^{M}\right)}{\widehat{\sigma}^{2}\left(X^{M}\right)}$. Based on the sample sets (19), (25), and (27), respectively, from the variables $\varepsilon^{M}, X^{M}$, and $Y^{M}$, the asymptotic distribution (43) is proven. Let us study first the law convergence of $\sqrt{n k}(\widehat{\alpha}-\alpha)=$ $\sqrt{n k}\left(\frac{\widehat{\sigma}\left(Y^{M}, X^{M}\right)}{\widehat{\sigma}^{2}\left(X^{M}\right)}-\alpha\right)$. By considering $Y_{j}^{M *}=\alpha X_{j}^{M *}+\gamma+\varepsilon_{j}^{M *}$ for all 
$j=1, \ldots, n k$ (see the $j$ th element of the sample sets $\varepsilon^{M *}$ in (19), $X^{M *}$ in (25), and $Y^{M *}$ in (27)), we obtain $\frac{\widehat{\sigma}\left(Y^{M}, X^{M}\right)}{\widehat{\sigma}^{2}\left(X^{M}\right)}=\alpha+\frac{\widehat{\sigma}\left(\varepsilon^{M}, X^{M}\right)}{\widehat{\sigma}^{2}\left(X^{M}\right)}$, and hence $\sqrt{n k}(\widehat{\alpha}-\alpha)=\sqrt{n k}\left(\frac{\widehat{\sigma}\left(Y^{M}, X^{M}\right)}{\widehat{\sigma}^{2}\left(X^{M}\right)}-\alpha\right)=\sqrt{n k} \frac{\widehat{\sigma}\left(\varepsilon^{M}, X^{M}\right)}{\widehat{\sigma}^{2}\left(X^{M}\right)}$. By adding $E\left(X^{M}\right)-E\left(X^{M}\right)$ in $\sqrt{n k} \widehat{\sigma}\left(\varepsilon^{M}, X^{M}\right)=(1 / \sqrt{n k}) \sum_{j=1}^{n k}\left(X_{j}^{M *}-\overline{X^{M *}}\right)$ $\left(\varepsilon_{j}^{M *}-\overline{\varepsilon^{M *}}\right)$ (with $\overline{X^{M *}}=\frac{1}{n k} \sum_{j=1}^{n k} X_{j}^{M *}$, and analogously $\overline{\varepsilon^{M *}}$ ), we conclude

$$
\begin{gathered}
\sqrt{n k} \widehat{\sigma}\left(\varepsilon^{M}, X^{M}\right)=\left(\frac{1}{\sqrt{n k}} \sum_{j=1}^{n k}\left(X_{j}^{M *}-E\left(X^{M}\right)\right) \varepsilon_{j}^{M *}\right)+ \\
\left(\left(E\left(X^{M}\right)-\overline{X^{M *}}\right) \frac{1}{\sqrt{n k}} \sum_{j=1}^{n k} \varepsilon_{j}^{M *}\right)=I_{1}+I_{2} .
\end{gathered}
$$

Since $\left\{\left(X_{j}^{M *}-E\left(X^{M}\right)\right) \varepsilon_{j}^{M *}\right\}_{j=1}^{n k}$ is a collection of i.i.d. real-valued variables with mean equal to 0 and variance equal to $\sigma^{2}\left(X^{M}\right) \sigma^{2}\left(\varepsilon^{M}\right)$, using the Central Limit Theorem, we can express $I_{1} \stackrel{\mathcal{L}}{\longrightarrow} N\left(0, \sigma^{2}\left(X^{M}\right) \sigma^{2}\left(\varepsilon^{M}\right)\right)$. An analogous reasoning to the variables $\left\{\varepsilon_{j}^{M *}\right\}_{j=1}^{n k}$ drives to $(1 / \sqrt{n k}) \sum_{j=1}^{n k} \varepsilon_{j}^{M *}$ $\stackrel{\mathcal{L}}{\longrightarrow} N\left(0, \sigma^{2}\left(\varepsilon^{M}\right)\right)$. Using the Strong Law of Large Numbers, we conclude that the expression $\left(E\left(X^{M}\right)-\overline{X^{M *}}\right)$ converges almost surely to zero. Thus, by applying the Slutzky Theorem, we can express $I_{2} \stackrel{P}{\rightarrow} 0$. The strong consistency of $\widehat{\sigma}^{2}\left(X^{M}\right)$ with respect to $\sigma^{2}\left(X^{M}\right)$ allows to use the Slutzky Theorem in $\sqrt{n k} \frac{\widehat{\sigma}\left(\varepsilon^{M}, X^{M}\right)}{\widehat{\sigma}^{2}\left(X^{M}\right)}$ finally obtain $\sqrt{n k}(\widehat{\alpha}-\alpha) \stackrel{\mathcal{L}}{\longrightarrow} N\left(0, \frac{\sigma^{2}\left(\varepsilon^{M}\right)}{\sigma^{2}\left(X^{M}\right)}\right)$.

The expression $\widehat{\beta}_{1}=\frac{\widehat{\sigma}\left(Y, X^{S}\right)}{\widehat{\sigma}^{2}\left(X^{S}\right)}$ in $(36)$ can be written as $\widehat{\beta}_{1}=\frac{\widehat{\sigma}\left(Y^{S}, X^{S}\right)}{\widehat{\sigma}^{2}\left(X^{S}\right)}$ (see the proof of Theorem 2). Also, based on Section 2.1, $\frac{\widehat{\sigma}\left(Y^{S}, X^{S}\right)}{\widehat{\sigma}^{2}\left(X^{S}\right)}$ is equal to $\frac{\widehat{\sigma}(\operatorname{spr} Y, \operatorname{spr} X)}{\widehat{\sigma}^{2}(\operatorname{spr} X)}$. The asymptotic distribution (44) is proven according to Section 2.1, and also the sample sets given in (20), (23), and (28) from the variables $\operatorname{spr} \varepsilon, \operatorname{spr} X$, and $\operatorname{spr} Y$, respectively. Let us study first the law convergence of $\sqrt{n k}\left(\widehat{\beta}_{1}-\beta\right)=\sqrt{n k}\left(\frac{\widehat{\sigma}(\operatorname{spr} Y, \operatorname{spr} X)}{\widehat{\sigma}^{2}(\operatorname{spr} X)}-\beta\right)$. Taking into account that $Y_{j}^{S p *}=\beta X_{j}^{S p *}+\varepsilon_{j}^{S p *}$ for all $j=1, \ldots, n k$ (see the $j$ th el- 
ement of the sets $\varepsilon^{S p *}, X^{S p *}$, and $Y^{S p *}$, respectively, given in (20), (23), and $(28)), \frac{\widehat{\sigma}(\operatorname{spr} Y, \operatorname{spr} X)}{\widehat{\sigma}^{2}(\operatorname{spr} X)}=\beta+\frac{\widehat{\sigma}(\operatorname{spr} \varepsilon, \operatorname{spr} X)}{\widehat{\sigma}^{2}(\operatorname{spr} X)}$ can be written. The rest of the proof process is analogous to the proof process of (43). Finally, we conclude $\sqrt{n k}\left(\widehat{\beta}_{1}-\beta\right) \stackrel{\mathcal{L}}{\longrightarrow} N\left(0, \frac{\sigma^{2}(\operatorname{spr} \varepsilon)}{\sigma^{2}(\operatorname{spr} X)}\right)$. Based on Section 2.1, we can express $\frac{\sigma^{2}(\operatorname{spr} \varepsilon)}{\sigma^{2}(\operatorname{spr} X)}=\frac{\sigma^{2}\left(\varepsilon^{S}\right)}{\sigma^{2}\left(X^{S}\right)}$, and hence $\sqrt{n k}\left(\widehat{\beta}_{1}-\beta\right) \stackrel{\mathcal{L}}{\longrightarrow} N\left(0, \frac{\sigma^{2}\left(\varepsilon^{S}\right)}{\sigma^{2}\left(X^{S}\right)}\right)$.

The inferential study of the model (1) can be developed on the basis of the asymptotic distributions of the old known estimators $\widehat{\gamma}$ in (37) and $\widehat{\delta}$ in (38).

Based on Theorem 3, the asymptotic variances of the old known estimators $\widehat{\alpha}$ and $\widehat{\beta}$ are $\operatorname{Var}(\widehat{\alpha})=\operatorname{Var}(\widehat{\alpha}-\alpha) \approx \frac{\sigma^{2}\left(\varepsilon^{M}\right)}{n k \sigma^{2}\left(X^{M}\right)}$ and $\operatorname{Var}(\widehat{\beta})=$ $\operatorname{Var}(\widehat{\beta}-\beta) \approx \operatorname{Var}\left(\widehat{\beta}_{1}\right)=\operatorname{Var}\left(\widehat{\beta}_{1}-\beta\right) \approx \frac{\sigma^{2}\left(\varepsilon^{S}\right)}{n k \sigma^{2}\left(X^{S}\right)}$, respectively. Also, based on the proof of Theorem 2 and the asymptotic variances, the asymptotic MSE of the old estimator $\widehat{\alpha}$ in $(35)$ is $M S E(\widehat{\alpha})=(E(\widehat{\alpha})-\alpha)^{2}+$ $\operatorname{Var}(\widehat{\alpha}) \approx\left(\frac{\sigma\left(\varepsilon^{M}, X^{M}\right)}{\sigma^{2}\left(X^{M}\right)}\right)^{2}+\frac{\sigma^{2}\left(\varepsilon^{M}\right)}{n k \sigma^{2}\left(X^{M}\right)}$, and the asymptotic MSE of $\widehat{\beta}$ in (36) is $\operatorname{MSE}(\widehat{\beta})=(E(\widehat{\beta})-\beta)^{2}+\operatorname{Var}(\widehat{\beta}) \approx\left(\frac{\sigma\left(\varepsilon^{S}, X^{S}\right)}{\sigma^{2}\left(X^{S}\right)}\right)^{2}+\frac{\sigma^{2}\left(\varepsilon^{S}\right)}{n k \sigma^{2}\left(X^{S}\right)}$. When $n k \rightarrow \infty$, we can express $M S E(\widehat{\alpha}) \approx 0$ and $\operatorname{MSE}(\widehat{\beta}) \approx 0$.

\subsection{Optimal Properties of the New Estimators}

In this section, under the conditions $n k \rightarrow \infty$ and $Y^{I}-Y \stackrel{\mathcal{L}}{\longrightarrow} \underline{0}$ (this means $Y^{I M}-Y^{M} \stackrel{\mathcal{L}}{\longrightarrow} \underline{0}$ and $\left.Y^{I S}-Y^{S} \stackrel{\mathcal{L}}{\longrightarrow} \underline{0}\right)$, we investigate the asymptotic unbiasedness, the strong consistency, the asymptotic distribution, the asymptotic variance, and the asymptotic MSE of each of the new estimators (39)-(42). The study of these properties is one of the innovations and novelties of this article.

Theorem 4. Let $X, Y$ be random intervals verifying a model (1), such that $\sigma\left(Y^{I}, X\right), \sigma(Y, X), \sigma\left(Y^{I M}, X^{M}\right), \sigma\left(Y^{M}, X^{M}\right), \sigma\left(Y^{I S}, X^{S}\right), \sigma\left(Y^{S}, X^{S}\right)<\infty$, and $0<\sigma^{2}\left(Y^{I}\right), \sigma^{2}(Y), \sigma^{2}(X), \sigma^{2}\left(Y^{I M}\right), \sigma^{2}\left(Y^{M}\right), \sigma^{2}\left(X^{M}\right), \sigma^{2}\left(Y^{I S}\right) \sigma^{2}\left(Y^{S}\right)$, $\sigma^{2}\left(X^{S}\right)<\infty$. Under the conditions $n k \rightarrow \infty$ and $Y^{I}-Y \stackrel{\mathcal{L}}{\longrightarrow} \underline{0}$, the new estimators are strongly consistent with respect to the parameters of the model (1). 
Proof. We have $\frac{\widehat{\sigma}\left(X^{M}, Y^{I}\right)}{\widehat{\sigma}^{2}\left(X^{M}\right)}=\frac{\widehat{\sigma}\left(X^{M}, Y^{I M}\right)}{\widehat{\sigma}^{2}\left(X^{M}\right)}$ in $(39)$ and $\frac{\widehat{\sigma}\left(X^{S}, Y^{I}\right)}{\widehat{\sigma}^{2}\left(X^{S}\right)}=\frac{\widehat{\sigma}\left(X^{S}, Y^{I S}\right)}{\widehat{\sigma}^{2}\left(X^{S}\right)}$ in (40), because the variables $Y^{I S}$ and $Y^{I M}$ in $Y^{I}=Y^{I S}+Y^{I M}$ are independent from the variables $X^{M}$ and $X^{S}$, respectively. Using the second condition, the models $Y^{I}=\alpha X^{M}+\beta X^{S}+\gamma[1 \pm 0]+\varepsilon, Y^{I M}=\alpha X^{M}+\gamma[1 \pm 0]+\varepsilon^{M}$, $Y^{I S}=\beta X^{S}+\varepsilon^{S}$, and the new estimators (see (39)-(42)) change to the models $Y=\alpha X^{M}+\beta X^{S}+\gamma[1 \pm 0]+\varepsilon, Y^{M}=\alpha X^{M}+\gamma[1 \pm 0]+\varepsilon^{M}, Y^{S}=\beta X^{S}+\varepsilon^{S}$, and the old known estimators (see (35)-(38)), respectively. Based on Theorem 1, the old known estimators are strongly consistent with respect to the parameters of the model (1). So, under the conditions, the new estimators are consistent estimators with respect to the parameters. This means $\widehat{\alpha} \stackrel{n k \rightarrow \infty}{\longrightarrow} \alpha, \widehat{\beta} \stackrel{n k \rightarrow \infty}{\longrightarrow} \beta, \widehat{\gamma} \stackrel{n k \rightarrow \infty}{\longrightarrow} \gamma$, and $\widehat{\delta} \stackrel{n k \rightarrow \infty}{\longrightarrow} \delta$ a.s. $-[\mathrm{P}]$.

Under the conditions $n k \rightarrow \infty$ and $Y^{I}-Y \stackrel{\mathcal{L}}{\longrightarrow} \underline{0}$, the new estimators like the old known estimators in Blanco-Fernández et al. (2011) are strongly consistent (see Theorem 4). Under the conditions, considering the proof of Theorem 4, we can conclude that the new estimators (39)-(42), respectively, are (asymptotically) equivalent to the old known estimators (35)-(38) in the strong consistency property. This means that under the conditions, in the mentioned property, none of the new estimators is absolutely superior to the corresponding old estimator. Hence, under the conditions, we conclude no absolute superiority of one of the sets of the old known and new estimators to another in the mentioned property.

Theorem 5. Let $X, Y$ be random intervals verifying a model (1), such that $0<\sigma^{2}\left(Y^{I}\right), \sigma^{2}(Y), \sigma^{2}(X)<\infty$, and $\sigma\left(X, Y^{I}\right), \sigma(X, Y)<\infty$. Under the conditions $n k \rightarrow \infty$ and $Y^{I}-Y \stackrel{\mathcal{L}}{\longrightarrow} \underline{0}$, the new estimators are asymptotically unbiased.

Proof. By considering Section 2.1, from $0<\sigma^{2}\left(Y^{I}\right), \sigma^{2}(Y), \sigma^{2}(X)<\infty$ and $\sigma\left(X, Y^{I}\right), \sigma(X, Y)<\infty$, we conclude, respectively, $0<\sigma^{2}\left(Y^{I M}\right), \sigma^{2}\left(Y^{I S}\right)$, $\sigma^{2}\left(Y^{M}\right), \sigma^{2}\left(Y^{S}\right), \sigma^{2}\left(X^{M}\right), \sigma^{2}\left(X^{S}\right)<\infty$ and $\sigma\left(X^{M}, Y^{I M}\right), \sigma\left(X^{S}, Y^{I S}\right)$, $\sigma\left(X^{M}, Y^{M}\right), \sigma\left(X^{S}, Y^{S}\right)<\infty$. Hence, considering Section 2.1 and the definition of the variable $Y=Y^{M}+Y^{S}$ in (2) (with $Y^{M}=\alpha X^{M}+\gamma[1 \pm 0]+\varepsilon^{M}$ and $\left.Y^{S}=\beta X^{S}+\varepsilon^{S}\right)$, we conclude $\sigma\left(X^{M}, \varepsilon^{M}\right), \sigma\left(X^{S}, \varepsilon^{S}\right)<\infty$ from $\sigma\left(X^{M}, Y^{M}\right), \sigma\left(X^{S}, Y^{S}\right)<\infty$ and $0<\sigma^{2}\left(X^{M}\right), \sigma^{2}\left(X^{S}\right)<\infty$. As a result, we will have $\sigma(X, \varepsilon)=\sigma\left(X^{M}, \varepsilon^{M}\right)+\sigma\left(X^{S}, \varepsilon^{S}\right)<\infty$. The new estimator $\widehat{\alpha}=\frac{\widehat{\sigma}\left(X^{M}, Y^{I}\right)}{\widehat{\sigma}^{2}\left(X^{M}\right)}=\frac{\widehat{\sigma}\left(X^{M}, Y^{I M}+Y^{I S}\right)}{\widehat{\sigma}^{2}\left(X^{M}\right)}$ in (39) can be expressed as 
$\widehat{\alpha}=\frac{\widehat{\sigma}\left(X^{M}, Y^{I M}\right)}{\widehat{\sigma}^{2}\left(X^{M}\right)}$, because the variables $X^{M}$ and $Y^{I S}$ are independent from each other. By considering the definition of the variable $Y^{I}$ in Section 3, we conclude that the old estimators (35) and (37) are equal to the new estimators (39) and (41), respectively, and the condition $Y^{I M}-Y^{M} \stackrel{\mathcal{L}}{\longrightarrow} \underline{0}$ is always confirmed. The expression $\frac{\widehat{\sigma}\left(X^{S}, Y^{I}\right)}{\widehat{\sigma}^{2}\left(X^{S}\right)}=\frac{\widehat{\sigma}\left(X^{S}, Y^{I M}+Y^{I S}\right)}{\widehat{\sigma}^{2}\left(X^{S}\right)}$ in (40) can be written as $\frac{\widehat{\sigma}\left(X^{S}, Y^{I S}\right)}{\widehat{\sigma}^{2}\left(X^{S}\right)}$, because the variable $X^{S}$ is independent from the variable $Y^{I M}$. Under the condition $Y^{I}-Y \stackrel{\mathcal{L}}{\longrightarrow} \underline{0}$, the linear models $Y^{I S}=\beta X^{S}+\varepsilon^{S}$, $Y^{I}=\alpha X^{M}+\beta X^{S}+\gamma[1 \pm 0]+\varepsilon$, and the new estimators (40) and (42) change to the linear models $Y^{S}=\beta X^{S}+\varepsilon^{S}, Y=\alpha X^{M}+\beta X^{S}+\gamma[1 \pm 0]+\varepsilon$, and the old known estimators (36) and (38), respectively. Based on the proof of Theorem 2, the old known estimators are asymptotically unbiased. Based on part of the results obtained from Theorem 4, the new estimators are asymptotically unbiased. Hence, under the conditions, the asymptotic unbiasedness of the new estimators can be concluded as $E(\widehat{\alpha})-\alpha \approx \frac{\sigma\left(X^{M}, \varepsilon^{M}\right)}{\sigma^{2}\left(X^{M}\right)} \approx 0$, $E(\widehat{\beta})-\beta \approx \frac{\sigma\left(X^{S}, \varepsilon^{S}\right)}{\sigma^{2}\left(X^{S}\right)} \approx 0, E(\widehat{\gamma})-\gamma=-E\left(\frac{\widehat{\sigma}\left(X^{M}, \varepsilon^{M}\right)}{\widehat{\sigma}^{2}\left(X^{M}\right)} \overline{X^{M *}}\right) \approx 0$, and $E(\widehat{\delta})-\delta \approx-E\left(\frac{\widehat{\sigma}\left(X^{S}, \varepsilon^{S}\right)}{\widehat{\sigma}^{2}\left(X^{S}\right)} \overline{X^{S p *}}\right) \approx 0$

Under the conditions $n k \rightarrow \infty$ and $Y^{I}-Y \stackrel{\mathcal{L}}{\longrightarrow} \underline{0}$, the new estimators like the old known estimators in Blanco-Fernández et al. (2011) are asymptotically unbiased (see Theorem 5). Under the conditions, based on the proof of Theorem 5, in the asymptotic unbiasedness property, the new estimators (40) and (42), respectively, are (asymptotically) equivalent to the old known estimators (36) and (38). So, under the conditions, none of the new estimators (39)-(42) is absolutely better than the corresponding old estimator in the mentioned property. Hence, in the mentioned property, we conclude no absolute superiority of one of the sets of the old known and new estimators to another under the conditions.

No study has been done about the asymptotic distributions, the asymptotic variances, and the asymptotic MSEs of the old known estimators in the mentioned paper. In the rest of this section, we investigate the asymptotic distributions, the asymptotic variances, and the asymptotic MSEs of the new estimators (39) and (40).

Theorem 6. Assume that $X, Y$ be random intervals verifying a model (1). 
Under the conditions $Y^{I}-Y \stackrel{\mathcal{L}}{\longrightarrow} \underline{0}$ and $n k \rightarrow \infty$, if $0<\sigma^{2}\left(Y^{I M}\right), \sigma^{2}\left(Y^{I S}\right)$, $\sigma^{2}\left(Y^{M}\right), \sigma^{2}\left(Y^{S}\right), \sigma^{2}\left(X^{M}\right), \sigma^{2}\left(X^{S}\right)<\infty, \sigma\left(X^{M}, Y^{I M}\right), \sigma\left(X^{M}, Y^{M}\right), \sigma($ $\left.X^{S}, Y^{I S}\right), \sigma\left(X^{S}, Y^{S}\right)<\infty, \sigma^{2}\left(X^{M}\right), \sigma^{2}\left(X^{S}\right) \nrightarrow 0$, and as the results of the assumptions, $0<\frac{\sigma^{2}\left(\varepsilon^{M}\right)}{\sigma^{2}\left(X^{M}\right)}, \frac{\sigma^{2}\left(\varepsilon^{S}\right)}{\sigma^{2}\left(X^{S}\right)}<\infty$, then

$$
\sqrt{n k}(\widehat{\alpha}-\alpha) \stackrel{\mathcal{L}}{\longrightarrow} N\left(0, \frac{\sigma^{2}\left(\varepsilon^{M}\right)}{\sigma^{2}\left(X^{M}\right)}\right),
$$

and

$$
\sqrt{n k}\left(\widehat{\beta}_{1}-\beta\right) \stackrel{\mathcal{L}}{\longrightarrow} N\left(0, \frac{\sigma^{2}\left(\varepsilon^{S}\right)}{\sigma^{2}\left(X^{S}\right)}\right),
$$

where $\widehat{\alpha}$ and $\widehat{\beta}_{1}=\frac{\widehat{\sigma}\left(Y^{I}, X^{S}\right)}{\widehat{\sigma}^{2}\left(X^{S}\right)}$ are the estimators proposed in (39) and (40), respectively.

There is not a unique asymptotic distribution for the new estimator $\widehat{\beta}$ in (40), which depends on min and max operators. Hence, using $\widehat{\beta}_{1}=\frac{\widehat{\sigma}\left(Y^{I}, X^{S}\right)}{\widehat{\sigma}^{2}\left(X^{S}\right)}$ as the partial information of (40), a valid asymptotic distribution is proposed. The distributions (45) and (46), respectively, are the asymptotic distributions of the estimators $\widehat{\alpha}$ in (39) and $\widehat{\beta}_{1}$.

Proof. Section 2.1 shows that if we have $0<\sigma^{2}\left(Y^{I M}\right), \sigma^{2}\left(Y^{I S}\right), \sigma^{2}\left(Y^{M}\right), \sigma^{2}$ $\left(Y^{S}\right), \sigma^{2}\left(X^{M}\right), \sigma^{2}\left(X^{S}\right)<\infty$ and $\sigma\left(X^{M}, Y^{I M}\right), \sigma\left(X^{S}, Y^{I S}\right), \sigma\left(X^{M}, Y^{M}\right)$, $\sigma\left(X^{S}, Y^{S}\right)<\infty$, then we can conclude $0<\sigma^{2}\left(Y^{I}\right), \sigma^{2}(Y), \sigma^{2}(X)<\infty$ and $\sigma\left(X, Y^{I}\right), \sigma(X, Y)<\infty$, respectively. Hence, considering Section 2.1, the definition of the variable $Y$ in (2), the assumptions of Theorem 6 , and the results of the assumptions, we conclude $0<\sigma^{2}(\varepsilon), \sigma^{2}\left(\varepsilon^{M}\right), \sigma^{2}\left(\varepsilon^{S}\right)<\infty$ and $\sigma(X, \varepsilon), \sigma\left(X^{M}, \varepsilon^{M}\right), \sigma\left(X^{S}, \varepsilon^{S}\right)<\infty$. As results, if we have $\sigma^{2}\left(X^{M}\right)$, $\sigma^{2}\left(X^{S}\right) \nrightarrow 0$, then we will have $0<\frac{\sigma^{2}\left(\varepsilon^{M}\right)}{\sigma^{2}\left(X^{M}\right)}, \frac{\sigma^{2}\left(\varepsilon^{S}\right)}{\sigma^{2}\left(X^{S}\right)}<\infty$.

In the proof of Theorem 5, we demonstrated that the old estimator $\widehat{\alpha}$ in (35) and the new estimator $\widehat{\alpha}$ in (39) are equal. Based on (43) in Theorem 3, we know $\sqrt{n k}\left(\frac{\widehat{\sigma}\left(Y, X^{M}\right)}{\widehat{\sigma}^{2}\left(X^{M}\right)}-\alpha\right)=\sqrt{n k}\left(\frac{\widehat{\sigma}\left(Y^{M}, X^{M}\right)}{\widehat{\sigma}^{2}\left(X^{M}\right)}-\alpha\right) \stackrel{\mathcal{L}}{\longrightarrow}$ $N\left(0, \frac{\sigma^{2}\left(\varepsilon^{M}\right)}{\sigma^{2}\left(X^{M}\right)}\right)$. Hence, the asymptotic distribution of the estimator (39) can be presented as $\sqrt{n k}(\widehat{\alpha}-\alpha)=\sqrt{n k}\left(\frac{\widehat{\sigma}\left(Y^{I}, X^{M}\right)}{\widehat{\sigma}^{2}\left(X^{M}\right)}-\alpha\right)=\sqrt{n k}\left(\frac{\widehat{\sigma}\left(Y^{I M}, X^{M}\right)}{\widehat{\sigma}^{2}\left(X^{M}\right)}\right.$ 
$-\alpha) \stackrel{\mathcal{L}}{\longrightarrow} N\left(0, \frac{\sigma^{2}\left(\varepsilon^{M}\right)}{\sigma^{2}\left(X^{M}\right)}\right)$

The distribution (46) is proven according to the sample sets given in (23), (20), (34), and (28) from the variables $\operatorname{spr} X, \operatorname{spr} \varepsilon, \operatorname{spr} Y^{I}$, and $\operatorname{spr} Y$, respectively. Under the conditions $Y^{I}-Y \stackrel{\mathcal{L}}{\longrightarrow} \underline{0}$ and $n k \rightarrow \infty$, the linear model $Y^{I S}=\beta X^{S}+\varepsilon^{S}$ and the estimator $\widehat{\beta}_{1}=\frac{\widehat{\sigma}\left(Y^{I}, X^{S}\right)}{\widehat{\sigma}^{2}\left(X^{S}\right)}=\frac{\widehat{\sigma}\left(Y^{I S}, X^{S}\right)}{\widehat{\sigma}^{2}\left(X^{S}\right)}$ in (40) change to the linear model $Y^{S}=\beta X^{S}+\varepsilon^{S}$ and the estimator $\widehat{\beta}_{1}=\frac{\widehat{\sigma}\left(Y, X^{S}\right)}{\widehat{\sigma}^{2}\left(X^{S}\right)}=$ $\frac{\widehat{\sigma}\left(Y^{S}, X^{S}\right)}{\widehat{\sigma}^{2}\left(X^{S}\right)}$ in $(36)$, respectively. The expression $\sqrt{n k}\left(\frac{\widehat{\sigma}\left(Y, X^{S}\right)}{\widehat{\sigma}^{2}\left(X^{S}\right)}-\beta\right) \stackrel{\mathcal{L}}{\longrightarrow}$ $N\left(0, \frac{\sigma^{2}\left(\varepsilon^{S}\right)}{\sigma^{2}\left(X^{S}\right)}\right)$ concluded in the proof of (44) of Theorem 3. Hence, we can write $\sqrt{n k}\left(\widehat{\beta}_{1}-\beta\right)=\sqrt{n k}\left(\frac{\widehat{\sigma}\left(Y^{I}, X^{S}\right)}{\widehat{\sigma}^{2}\left(X^{S}\right)}-\beta\right) \stackrel{\mathcal{L}}{\longrightarrow} N\left(0, \frac{\sigma^{2}\left(\varepsilon^{S}\right)}{\sigma^{2}\left(X^{S}\right)}\right)$ based on the conditions.

Under the conditions $n k \rightarrow \infty$ and $Y^{I}-Y \stackrel{\mathcal{L}}{\longrightarrow} \underline{0}$, considering the proof of Theorem 6, we can conclude that the asymptotic distribution (46) is (asymptotically) equivalent to the asymptotic distribution (44), and the asymptotic distribution (45) is the same the asymptotic distribution (43).

Based on Theorem 6, the asymptotic variances of (39) and (40) are $\operatorname{Var}(\widehat{\alpha})=\operatorname{Var}(\widehat{\alpha}-\alpha) \approx \frac{\sigma^{2}\left(\varepsilon^{M}\right)}{n k \sigma^{2}\left(X^{M}\right)}$ and $\operatorname{Var}(\widehat{\beta})=\operatorname{Var}(\widehat{\beta}-\beta) \approx \operatorname{Var}\left(\widehat{\beta}_{1}\right)$ $=\operatorname{Var}\left(\widehat{\beta}_{1}-\beta\right) \approx \frac{\sigma^{2}\left(\varepsilon^{S}\right)}{n k \sigma^{2}\left(X^{S}\right)}$. Based on $\operatorname{Var}(\widehat{\alpha}), \operatorname{Var}(\widehat{\beta})$, and the proof of Theorem 5, the asymptotic MSEs of (39) and (40) are, respectively, $M S E(\widehat{\alpha})=(E(\widehat{\alpha})-\alpha)^{2}+\operatorname{Var}(\widehat{\alpha}) \approx\left(\frac{\sigma\left(\varepsilon^{M}, X^{M}\right)}{\sigma^{2}\left(X^{M}\right)}\right)^{2}+\frac{\sigma^{2}\left(\varepsilon^{M}\right)}{n k \sigma^{2}\left(X^{M}\right)}$ and $M S E(\widehat{\beta})$ $=(E(\widehat{\beta})-\beta)^{2}+\operatorname{Var}(\widehat{\beta}) \approx\left(\frac{\sigma\left(\varepsilon^{S}, X^{S}\right)}{\sigma^{2}\left(X^{S}\right)}\right)^{2}+\frac{\sigma^{2}\left(\varepsilon^{S}\right)}{n k \sigma^{2}\left(X^{S}\right)}$. We can conclude $\operatorname{MSE}(\widehat{\alpha}) \approx 0$ and $\operatorname{MSE}(\widehat{\beta}) \approx 0$ when $n k \rightarrow \infty$.

Under the conditions $n k \rightarrow \infty$ and $Y^{I}-Y \stackrel{\mathcal{L}}{\longrightarrow} \underline{0}$, considering the proofs of Theorems 2, 3, 5, and 6, we can conclude that $\operatorname{Var}(\widehat{\beta}$ in $(36))$ and $M S E(\widehat{\beta}$ in $(36))$, respectively, are (asymptotically) equivalent to $\operatorname{Var}(\widehat{\beta}$ in $(40))$ and $M S E(\widehat{\beta}$ in (40)). So, $\operatorname{Var}(\widehat{\beta}$ in $(36))$ and $M S E(\widehat{\beta}$ in $(36))$, respectively, are not always less than $\operatorname{Var}(\widehat{\beta}$ in $(40))$ and $M S E(\widehat{\beta}$ in $(40))$. 
Also, we have $\operatorname{Var}(\widehat{\alpha}$ in (35)) $=\operatorname{Var}(\widehat{\alpha}$ in (39)) and $M S E(\widehat{\alpha}$ in (35)) $=$ $M S E(\widehat{\alpha}$ in $(39))$.

Based on the asymptotic distributions of the estimators (41) and (42), the inferential study of the model (1) can be developed.

\section{Studies}

\subsection{Simulation Studies}

Based on the new structure of MCR in Section 3, a researcher selects singlevalued samples within the random intervals of interval-valued variables. However, based on other sampling methods from interval variables, the researcher selects single-valued samples from mid variables and spr variables. Under the conditions $n k \rightarrow \infty$ and $Y^{I}-Y \stackrel{\mathcal{L}}{\longrightarrow} \underline{0}$, the empirical behaviors of the estimators (see Sections 4.1 and 4.2) are investigated when the parameters of the model (1) have known quantities. In this section, based on Section 3 , we consider $n$ and $k$, respectively, as the number of the simulations (iterations) and observations. The values calculated for the estimators $\widehat{\alpha^{b}}, \widehat{\beta^{b}}$, $\widehat{\gamma^{b}}$, and $\widehat{\delta^{b}}, b=1, \ldots, n$, in each of Sections 4.1 and 4.2 are denoted by the estimates $\widehat{\alpha_{*}^{b}}, \widehat{\beta_{*}^{b}}, \widehat{\gamma_{*}^{b}}$, and $\widehat{\delta}_{*}^{b}$, respectively. The vector of $n$ values for the estimate of each parameter of the model (1) will be defined as an empirical distribution of the corresponding estimator close to the theoretical one, because $n$ is large enough. Based on $n$ iterations, $\widehat{E}(\widehat{\Xi})=\left(\sum_{b=1}^{n} \widehat{\Xi_{*}^{b}}\right) / n$ and $\widehat{M S E}(\widehat{\Xi})=\left(\sum_{b=1}^{n}\left(\widehat{\Xi_{*}^{b}}-\Xi\right)^{2}\right) / n$ are calculated, respectively, as the estimated mean value and the estimated mean squared error of an estimator $\widehat{\Xi}$ from the empirical distribution, as empirical approximations of the theoretical ones. By considering $n$ repetitions, the mean square error of the estimated model (1) based on the sets of the old known and new estimates of the set of the parameters, respectively, is computed as

$$
M S E M O=\frac{\sum_{b=1}^{n}\left(\frac{\sum_{i=1}^{k}\left(Y_{i}^{M * b}-\widehat{Y}_{i}^{M * b}\right)^{2}}{k}\right)}{n}+\frac{\sum_{b=1}^{n}\left(\frac{\sum_{i=1}^{k}\left(Y_{i}^{S p * b}-\widehat{Y}_{i}^{S p * b}\right)^{2}}{k}\right)}{n}
$$

and 
$M S E M N=\frac{\sum_{b=1}^{n}\left(\frac{\sum_{i=1}^{k}\left(Y_{i}^{I M * b}-\widehat{Y}_{i}^{I M * b}\right)^{2}}{k}\right)}{n}+\frac{\sum_{b=1}^{n}\left(\frac{\sum_{i=1}^{k}\left(Y_{i}^{I S p * b}-\widehat{Y}_{i}^{I S p * b}\right)^{2}}{k}\right)}{n}$, where $Y_{i}^{M * b}, Y_{i}^{S p * b}, Y_{i}^{I M * b}$, and $Y_{i}^{I S p * b}$, respectively, are the $i^{\text {th }}$ element of the sample sets (11), (12), (17), and (18), $i=1, \ldots, k$ and $b=1, \ldots, n$. In the expression $M S E M O$, based on the old known estimates $\widehat{\alpha_{*}^{b}}, \widehat{\beta_{*}^{b}}, \widehat{\gamma_{*}^{b}}$, and $\widehat{\delta_{*}^{b}}$ calculated from the estimators in Section 4.1, $\widehat{Y}_{i}^{M * b}=\widehat{\alpha_{*}^{b}} X_{i}^{M * b}+\widehat{\gamma}_{*}^{b}$ and $\widehat{Y}_{i}^{S p * b}=\widehat{\beta}_{*}^{b} X_{i}^{S p * b}+\widehat{\delta}_{*}^{b}$ are defined, where $X_{i}^{M * b}$ and $X_{i}^{S p * b}$ are the $i^{t h}$ element of the sample sets (9) and (7), respectively. In the expression $M S E M N$, under the conditions, based on the new estimates $\widehat{\alpha_{*}^{b}}, \widehat{\beta_{*}^{b}}, \widehat{\gamma_{*}^{b}}$, and $\widehat{\delta_{*}^{b}}$ obtained from the estimators in Section 4.2, $\widehat{Y}_{i}^{I M * b}=\widehat{\alpha_{*}^{b}} X_{i}^{M * b}+\widehat{\gamma}_{*}^{b}$ and $\widehat{Y}_{i}^{I S p * b}=$ $\widehat{\beta}_{*}^{b} X_{i}^{S p * b}+\widehat{\delta_{*}^{b}}$ are introduced. In this section, $n=1000$ and $k=5,50,500$ (the first condition is fulfilled) are considered.

Some LMs with the structure of the model (1) are studied in this section. Different parameters are investigated in the LMs. In the models, the variables come from different distributions. The LMs, taking into account that the $s p r$ variables have to be non-negative, are provided.

Model $\mathbf{M}_{\mathbf{1}}$ : Let mid $X \sim \mathrm{N}(0,1)$, mid $\varepsilon \sim \mathrm{N}(0,0.2)$, and $\operatorname{spr} X, \operatorname{spr} \varepsilon \sim \chi_{1}^{2}$ be random variables, and let $Y$ be a random interval defined by means of the linear model

$$
Y=3 X^{M}-2 X^{S}+5[1 \pm 0]+\varepsilon=3 X^{M}+2 X^{S}+5[1 \pm 0]+\varepsilon .
$$

From the distribution considered for $\operatorname{spr} \varepsilon$, it can be shown that $\delta=1$, and hence $B=[\gamma \pm \delta]=[4,6]$.

Model $\mathbf{M}_{\mathbf{2}}$ : Let $\operatorname{mid} \varepsilon \sim \mathrm{N}(0,9), \operatorname{mid} X \sim \mathrm{N}(0,3), \operatorname{spr} \varepsilon \sim \chi_{4}^{2}$ and $\operatorname{spr} X \sim$ $\chi_{3}^{2}$ be random variables, and let the interval linear model between $X$ and $Y$ be

$$
Y=3 X^{M}-2 X^{S}+5[1 \pm 0]+\varepsilon=3 X^{M}+2 X^{S}+5[1 \pm 0]+\varepsilon .
$$

We can conclude $\delta=4$ and $B=[1,9]$ from the distribution of $\operatorname{spr} \varepsilon$.

Model $\mathbf{M}_{3}$ : Let $\operatorname{mid} X \sim \Gamma(1,50), \operatorname{mid} \varepsilon \sim \operatorname{Exp}(3000), \operatorname{spr} X \sim \operatorname{Beta}(0.7,0.7)$, 
Table 1. Empirical validation of the old known estimators with respect to the parameters of the model (1).

\begin{tabular}{cccccccccc}
\hline \hline \multirow{2}{*}{ Model } & $k$ & $\widehat{E}(\widehat{\alpha})$ & $\widehat{M S E}(\widehat{\alpha})$ & $\widehat{E}(\widehat{\beta})$ & $\widehat{M S E}(\widehat{\beta})$ & $\widehat{E}(\widehat{\gamma})$ & $\widehat{M S E}(\widehat{\gamma})$ & $\widehat{E}(\widehat{\delta})$ & $\widehat{M S E}(\widehat{\delta})$ \\
\hline \multirow{3}{*}{$M_{1}$} & 5 & 3.002162 & $1.0172 \mathrm{e}-01$ & 1.730412 & $4.6368 \mathrm{e}-01$ & 5.008412 & $5.2529 \mathrm{e}-02$ & 0.974601 & $2.1629 \mathrm{e}-01$ \\
& 50 & 2.999661 & $4.3119 \mathrm{e}-03$ & 1.929245 & $4.5035 \mathrm{e}-02$ & 5.002541 & $4.0134 \mathrm{e}-03$ & 1.001770 & $2.1064 \mathrm{e}-02$ \\
& 500 & 3.000241 & $4.0934 \mathrm{e}-04$ & 1.940257 & $4.8875 \mathrm{e}-03$ & 4.999633 & $4.0328 \mathrm{e}-04$ & 0.998545 & $2.5570 \mathrm{e}-03$ \\
\hline \multirow{4}{*}{$M_{2}$} & 5 & 3.069161 & 1.6383171 & 1.826449 & $5.2137 \mathrm{e}-01$ & 5.039471 & 2.8635722 & 3.968221 & 1.9177650 \\
& 50 & 2.982802 & $6.0798 \mathrm{e}-02$ & 1.956279 & $2.4740 \mathrm{e}-02$ & 5.020446 & $1.8093 \mathrm{e}-01$ & 3.987342 & $1.6474 \mathrm{e}-01$ \\
& 500 & 3.012075 & $6.7971 \mathrm{e}-04$ & 1.987883 & $2.1121 \mathrm{e}-03$ & 4.988527 & $1.9421 \mathrm{e}-02$ & 4.013026 & $1.7294 \mathrm{e}-02$ \\
\hline \multirow{3}{*}{$M_{3}$} & 5 & 5.000469 & $4.1386 \mathrm{e}-04$ & 0.805601 & $1.5263 \mathrm{e}-01$ & $3.399 \mathrm{e}-04$ & $2.3939 \mathrm{e}-07$ & 0.496992 & $1.3734 \mathrm{e}-01$ \\
& 50 & 4.999887 & $6.3810 \mathrm{e}-06$ & 0.870884 & $6.0659 \mathrm{e}-02$ & $3.371 \mathrm{e}-04$ & $1.1855 \mathrm{e}-07$ & 0.502816 & $1.0669 \mathrm{e}-02$ \\
& 500 & 4.999975 & $5.9320 \mathrm{e}-07$ & 0.880639 & $5.6996 \mathrm{e}-02$ & $3.341 \mathrm{e}-04$ & $1.1205 \mathrm{e}-07$ & 0.500663 & $1.1646 \mathrm{e}-03$ \\
\hline \multirow{3}{*}{$M_{4}$} & 5 & 0.999108 & $1.1116 \mathrm{e}-02$ & 2.958663 & $1.8021 \mathrm{e}-01$ & 1.999715 & $3.6253 \mathrm{e}-02$ & 0.822683 & $6.4379 \mathrm{e}-02$ \\
& 50 & 1.000331 & $4.3875 \mathrm{e}-05$ & 3.000027 & $1.1697 \mathrm{e}-02$ & 2.000143 & $1.7053 \mathrm{e}-03$ & 0.832330 & $4.9334 \mathrm{e}-03$ \\
& 500 & 1.000001 & $4.9336 \mathrm{e}-07$ & 3.000013 & $1.1349 \mathrm{e}-03$ & 2.000126 & $1.6846 \mathrm{e}-04$ & 0.833693 & $5.0385 \mathrm{e}-04$ \\
\hline
\end{tabular}

and $\operatorname{spr} \varepsilon \sim \chi_{0.25}^{2}$ be random variables, and let $Y$ be

$$
Y=5 X^{M}-X^{S}+\varepsilon=5 X^{M}+X^{S}+\varepsilon .
$$

By considering the distribution of $\operatorname{spr} \varepsilon$, we conclude $\delta=0.5$, and thus $B=[-0.5,0.5]$.

Model $\mathbf{M}_{4}$ : Let $\operatorname{mid} X \sim \operatorname{Cauchy}(1,1), \operatorname{mid} \varepsilon \sim \mathrm{U}(-0.5,0.5), \operatorname{spr} X \sim$ $\mathrm{F}(100,200,10)$, and $\operatorname{spr} \varepsilon \sim \operatorname{Beta}(3,0.6)$ be random variables, and the interval variable $Y$ is defined as

$$
Y=X^{M}-3 X^{S}+2[1 \pm 0]+\varepsilon=X^{M}+3 X^{S}+2[1 \pm 0]+\varepsilon .
$$

By considering the distribution of $\operatorname{spr} \varepsilon$, we can show that $\delta=\frac{3}{3.6}$ and $B=$ $\left[2-\left(\frac{3}{3.6}\right), 2+\left(\frac{3}{3.6}\right)\right]$.

We use $G \sim \mathrm{U}(-0.005,0.005)$ (see Section 3) for calculating the new estimates of the parameters of models $M_{2}$ and $M_{4}$ in Table 2. Also, the new estimates of the parameters of models $M_{1}$ and $M_{3}$, respectively, are calculated using $G \sim \mathrm{U}(-0.0005,0.0005)$ and $G \sim \mathrm{U}(-0.00005,0.00005)$ in the table. Hence, the realization of the condition $Y^{I}-Y \stackrel{\mathcal{L}}{\longrightarrow} \underline{0}$ for all models is concluded.

Under the conditions $n k \rightarrow \infty$ and $Y^{I}-Y \stackrel{\mathcal{L}}{\longrightarrow} \underline{0}$, Tables 1 and 2 show the proximity of the old known and new estimates to the corresponding parame- 
Table 2. Empirical validation of the new estimators with respect to the parameters of the model (1).

\begin{tabular}{cccccccccc}
\hline \hline \multirow{2}{*}{ Model } & $k$ & $\widehat{E}(\widehat{\alpha})$ & $\widehat{M S E}(\widehat{\alpha})$ & $\widehat{E}(\widehat{\beta})$ & $\widehat{M S E}(\widehat{\beta})$ & $\widehat{E}(\widehat{\gamma})$ & $\widehat{M S E}(\widehat{\gamma})$ & $\widehat{E}(\widehat{\delta})$ & $\widehat{M S E}(\widehat{\delta})$ \\
\hline \multirow{3}{*}{$M_{1}$} & 5 & 3.002162 & $1.0172 \mathrm{e}-01$ & 1.732566 & $4.5965 \mathrm{e}-01$ & 5.008412 & $5.2529 \mathrm{e}-02$ & 0.975107 & $2.1576 \mathrm{e}-01$ \\
& 50 & 2.999661 & $4.3119 \mathrm{e}-03$ & 1.943085 & $3.7823 \mathrm{e}-02$ & 5.002541 & $4.0134 \mathrm{e}-03$ & 1.001380 & $2.0311 \mathrm{e}-02$ \\
& 500 & 3.000241 & $4.0934 \mathrm{e}-04$ & 1.971457 & $4.1111 \mathrm{e}-03$ & 4.999633 & $4.0328 \mathrm{e}-04$ & 0.998606 & $2.0114 \mathrm{e}-03$ \\
\hline \multirow{3}{*}{$M_{2}$} & 5 & 3.069161 & 1.6383171 & 1.826418 & $5.2144 \mathrm{e}-01$ & 5.039471 & 2.8635722 & 3.968214 & 1.9178040 \\
& 50 & 2.982802 & $6.0798 \mathrm{e}-02$ & 1.956268 & $2.4742 \mathrm{e}-02$ & 5.020446 & $1.8093 \mathrm{e}-01$ & 3.987341 & $1.6475 \mathrm{e}-01$ \\
& 500 & 3.012075 & $6.7971 \mathrm{e}-04$ & 1.987882 & $2.1124 \mathrm{e}-03$ & 4.988527 & $1.9421 \mathrm{e}-02$ & 4.013030 & $1.7295 \mathrm{e}-02$ \\
\hline \multirow{3}{*}{$M_{3}$} & 5 & 5.000469 & $4.1386 \mathrm{e}-04$ & 0.805728 & $1.5261 \mathrm{e}-01$ & $3.399 \mathrm{e}-04$ & $2.3939 \mathrm{e}-07$ & 0.496999 & $1.3733 \mathrm{e}-01$ \\
& 50 & 4.999887 & $6.3810 \mathrm{e}-06$ & 0.876845 & $5.5623 \mathrm{e}-02$ & $3.371 \mathrm{e}-04$ & $1.1855 \mathrm{e}-07$ & 0.502723 & $1.0650 \mathrm{e}-02$ \\
& 500 & 4.999975 & $5.9320 \mathrm{e}-07$ & 0.944114 & $4.7068 \mathrm{e}-02$ & $3.341 \mathrm{e}-04$ & $1.1205 \mathrm{e}-07$ & 0.500630 & $1.1448 \mathrm{e}-03$ \\
\hline & 5 & 0.999108 & $1.1116 \mathrm{e}-02$ & 2.958495 & $1.8047 \mathrm{e}-01$ & 1.999715 & $3.6253 \mathrm{e}-02$ & 0.822507 & $6.4394 \mathrm{e}-02$ \\
$M_{4}$ & 50 & 1.000331 & $4.3875 \mathrm{e}-05$ & 3.000064 & $1.1706 \mathrm{e}-02$ & 2.000143 & $1.7053 \mathrm{e}-03$ & 0.832319 & $4.9344 \mathrm{e}-03$ \\
& 500 & 1.000001 & $4.9336 \mathrm{e}-07$ & 3.000019 & $1.1363 \mathrm{e}-03$ & 2.000126 & $1.6846 \mathrm{e}-04$ & 0.833701 & $5.0395 \mathrm{e}-04$ \\
\hline
\end{tabular}

ters in models $M_{1}-M_{4}$ as $k$ increases. This means the empirical validation of the old known and new estimators of the model (1) under the conditions. We know that if $k$ increases, then $n k=1000 k$ increases. Under the conditions, in Tables 1 and 2, based on the reported experimental results for $n=1000$ random samples (see (3)-(18) in Section 3) of different size $k$ from models $M_{1}-M_{4}$, the following conclusions can be expressed: First, the empirical asymptotic unbiasedness of the old known and new estimators are manifest when $k$ increases. In the tables, for instance, under the conditions, we see the empirical asymptotic unbiasedness of the old known and new estimators for model $M_{2}$ when $k$ or $n k=1000 k$ increases. Second, in Tables 1 and 2, as $k$ increases, $\widehat{M S E}(\widehat{\alpha}), \widehat{M S E}(\widehat{\beta}), \widehat{M S E}(\widehat{\gamma})$, and $\widehat{M S E}(\widehat{\delta})$ go to zero. This means the empirical consistency of the old known and new estimators under the conditions.

The study of the empirical validation (the empirical asymptotic unbiasedness and the empirical consistency) of the new estimators is the empirical novelty of this paper.

We concluded in the proof of Theorem 5 that the old known estimators (35) and (37) are equal to the new estimators (39) and (41), respectively. Hence, these results are repeated in empirical consistency and empirical asymptotic unbiasedness, so that the values of the third, fourth, seventh, and eighth columns of Table 1 are equal to the corresponding values in the third, fourth, seventh, and eighth columns of Table 2. 
Table 3. The empirical comparison between the old known and new estimation methods of the model (1).

\begin{tabular}{|c|c|c|c|c|c|c|c|}
\hline Model & $k$ & $\begin{array}{c}\Delta=\widehat{M S E}(\widehat{\alpha})+\widehat{M S E}(\widehat{\beta})+ \\
\widehat{M S E}(\widehat{\gamma})+\widehat{M S E}(\widehat{\delta}) \\
\text { from Table } 1\end{array}$ & $\begin{array}{c}\mathfrak{L}=\widehat{M S E}(\widehat{\alpha})+\widehat{M S E}(\widehat{\beta})+ \\
\widehat{M S E}(\widehat{\gamma})+\widehat{M S E}(\widehat{\delta}) \\
\text { from Table } 2\end{array}$ & $M S E M O$ & $M S E M N$ & $\frac{\mathfrak{L}}{\Delta}$ & $\frac{M S E M N}{M S E M O}$ \\
\hline \multirow{3}{*}{$M_{1}$} & 5 & 0.8342191 & 0.8296590 & 2.989085 & 2.966100 & 0.99453 & 0.99231 \\
\hline & 50 & 0.0744243 & 0.0664593 & 2.383716 & 2.282250 & 0.89298 & 0.95743 \\
\hline & 500 & 0.0082571 & 0.0069351 & 2.268857 & 2.004409 & 0.83989 & 0.88344 \\
\hline \multirow{3}{*}{$M_{2}$} & 5 & 6.9410240 & 6.9411333 & 21.80141 & 21.80247 & 1.00002 & 1.00005 \\
\hline & 50 & 0.4312081 & 0.4312201 & 17.07415 & 17.07425 & 1.00003 & 1.00001 \\
\hline & 500 & 0.0395068 & 0.0395081 & 16.96112 & 16.96113 & 1.00003 & 1.000001 \\
\hline \multirow{3}{*}{$M_{3}$} & 5 & 0.2903841 & 0.2903541 & 1.907982 & 1.907709 & 0.99990 & 0.99986 \\
\hline & 50 & 0.0713345 & 0.0662795 & 1.546166 & 1.544810 & 0.92913 & 0.99912 \\
\hline & 500 & 0.0581609 & 0.0482135 & 1.526243 & 1.499192 & 0.82897 & 0.98228 \\
\hline \multirow{3}{*}{$M_{4}$} & 5 & 0.2919582 & 0.2922334 & 0.350045 & 0.351219 & 1.00094 & 1.00335 \\
\hline & 50 & 0.0183799 & 0.0183893 & 0.129875 & 0.129930 & 1.00051 & 1.00042 \\
\hline & 500 & 0.0018078 & 0.0018091 & 0.115237 & 0.115270 & 1.00072 & 1.00029 \\
\hline
\end{tabular}

Under the conditions, in empirical consistency and empirical asymptotic unbiasedness, Tables 1 and 2 show generally that each of the new estimators (39)-(42) is (asymptotically) equivalent to the corresponding old estimator. For models $M_{1}$ and $M_{3}$, under the conditions, the comparison between the values of Tables 1 and 2 shows that the new estimators (40) and (42), respectively, are superior to the old known estimators (36) and (38) in the empirical asymptotic properties. For model $M_{4}$, under the conditions, in the empirical properties, the comparison between the mentioned tables shows the superiority of the old estimators (36) and (38).

Under the conditions, for each $k$, in the empirical asymptotic properties, the comparison between the values of Tables 1 and 2 shows the superiority of the new estimators (39)-(42) for the parameters of models $M_{1}$ and $M_{3}$, and also the superiority of the old known estimators (35)-(38) for the parameters of model $M_{4}$.

For instance, under the conditions $n k \rightarrow \infty$ and $Y^{I}-Y \stackrel{\mathcal{L}}{\longrightarrow} \underline{0}$, Figure 1 illustrates empirically the consistency of the estimators (35), (36), (39), and (40) of model $M_{1}$ based on $n=1000$ simulated samples of $k$ observations (see (3)-(18) in Section 3) as $k$ (or $n k=1000 k$ ) increases. This means that $\widehat{M S E}(\widehat{\alpha}$ in $(35)), \widehat{M S E}(\widehat{\beta}$ in (36)), $\widehat{M S E}(\widehat{\alpha}$ in (39)), and $\widehat{M S E}(\widehat{\beta}$ in $(40))$ go to zero as $k$ increases. Because in all situations, the boxes reduce the spread around the true value of the corresponding parame- 

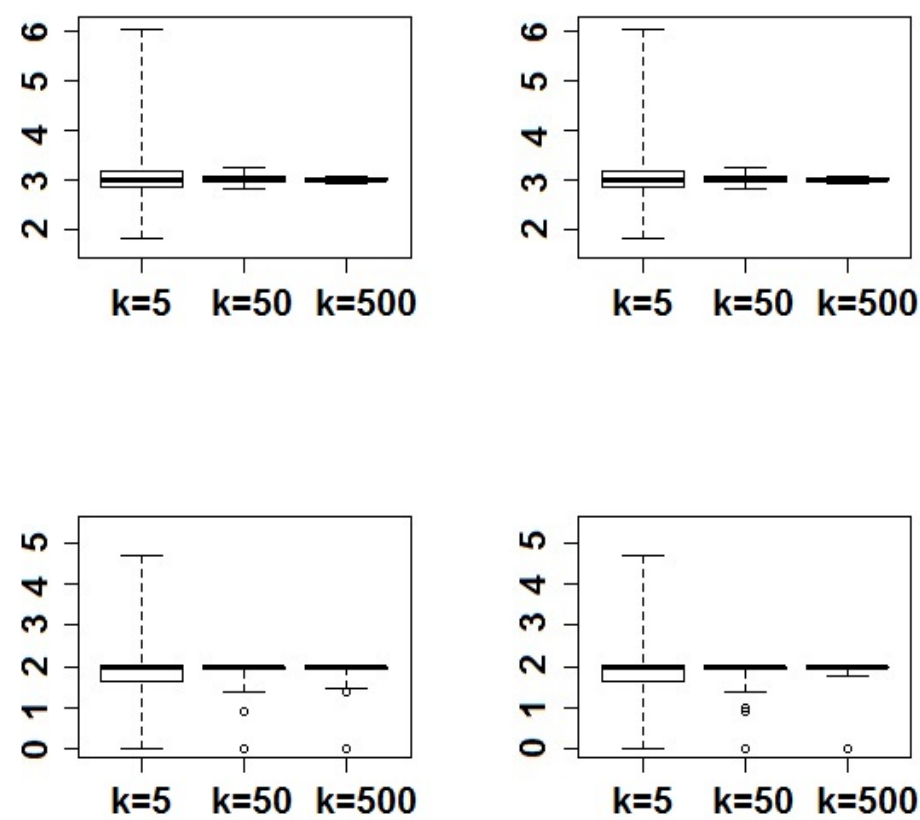

Figure 1. Box plot for the old known and new estimators of the parameter $\alpha$ (top left and top right, respectively) and the old known and new estimators of the parameter $\beta$ (bottom left and bottom right, respectively) for model $M_{1}$.

ter as the number of observations $k$ increases. Figure 1 illustrates the superiority of the new estimator $\widehat{\beta}$ in (40) to the old known estimator $\widehat{\beta}$ in (36) in empirical consistency when $k=500$ (or when $k$ increases) and $n=1000$. Since the estimators (35) and (39) are equal, hence the plots provided in Figure 1 for the old known and new estimators of the parameter $\alpha$ are the same.

Table 3 is provided under the conditions. As $k$ (or $n k=1000 k$ ) increases, the values of the third and fourth columns of Table 3 for each of models $M_{1^{-}}$ $M_{4}$ go to zero. This means the empirical consistency of the sets of the old known and new estimators of the model (1) under the conditions. Also, the values of the fifth and sixth columns of the table for each model decrease as $k$ increases. This means that under the conditions, the old known and 
new estimation methods, respectively, in Sections 4.1 and 4.2 are suitable. Table 3 shows that under the conditions, the new estimators in Section 4.2 are superior to the old known estimators in Section 4.1 (or the same the estimators presented in Blanco-Fernández et al. , 2011) for the parameters of models $M_{1}$ and $M_{3}$ in empirical consistency, because we have $\frac{\mathfrak{L}}{\Delta}<1$ for each $k$. Also, in empirical consistency, for each $k$, the seventh column of Table 3 shows the superiority of the old known estimators for the parameters of model $M_{4}$, because we have $\frac{\mathfrak{L}}{\Delta}>1$. For each $k$, the ratio $\frac{\mathfrak{L}}{\Delta}$ in Table 3 is (asymptotically) equivalent to one for model $M_{2}$. This means that under the conditions, in empirical consistency, none of the sets of the estimators is superior to the other one. Hence, under the conditions, the new estimators in Section 4.2 are superior to the old known estimators in Section 4.1 for the parameters of some LMs with the structure of the model (1) in empirical consistency. All the results provided for Table 3 will be confirmed when the last column of Table 3 instead of the seventh column of the table is investigated.

Overall, under the conditions, the results demonstrate well that for LMs with the structure of (1), the distributions of the variables $\operatorname{spr} \varepsilon$ and $\operatorname{spr} X$ are simultaneously effective in choosing a better set of the estimators from the two sets (see models $M_{1}$ and $M_{2}$ ). Table 3 shows no the absolute superiority of one of the sets of the old known and new estimators to another under the conditions.

\subsection{A Real-Life Case Study}

To motivate the methodology, the analysis of the linear relationship between the fluctuation of the systolic and diastolic blood pressure for the 100 patients hospitalized in a hospital is considered. Values of the blood pressures for each patient are measured at different points in a day. Therefore, the range of variation of each pressure over one day can be expressed using a real compact interval. The range of variation of the pressures of each patient on a concrete day are recorded as $X=$ "range of variation of the systolic blood pressure up to one decimal place over a day" in Table 4 or Table $5, Y^{I}=$ "range of variation of the diastolic blood pressure up to three decimal places over the same day" in Table 4, and $Y=$ "range of variation of the diastolic blood pressure up to one decimal place over the same day" in Table 5. If inf $Y^{I}$ and $\sup Y^{I}$ in Table 4 are rounded up to one decimal place, then we will have, respectively, $\inf Y$ and $\sup Y$ in Table 5. Diastolic blood pressure data 
Table 4. Data set on the range of variation of blood pressures of 100 patients.

\begin{tabular}{|c|c|c|c|c|c|c|c|}
\hline$X$ & $Y^{I}$ & $X$ & $Y^{I}$ & $X$ & $Y^{I}$ & $X$ & $Y^{I}$ \\
\hline$[15.0,20.5]$ & {$[7.353,12.547]$} & {$[8.6,14.5]$} & {$[4.663,9.137]$} & {$[13.8,19.9]$} & {$[4.554,9.146]$} & {$[13.2,17.9]$} & {$[5.462,9.538]$} \\
\hline$[10.5,20.8]$ & {$[6.164,11.536]$} & {$[9.0,17.0]$} & {$[4.351,11.349]$} & {$[8.2,16.5]$} & {$[3.579,9.321]$} & {$[10.0,21.6]$} & {$[4.259,10.241]$} \\
\hline$[11.5,20.1]$ & {$[6.660,13.140]$} & {$[8.5,16.8]$} & {$[4.151,10.049]$} & {$[9.3,15.5]$} & {$[5.490,10.610]$} & {$[8.5,17.2]$} & {$[3.652,8.448]$} \\
\hline$[11.3,17.0]$ & {$[5.594,10.706]$} & {$[11.4,18.1]$} & {$[3.998,8.802]$} & {$[12.6,17.6]$} & {$[5.161,9.639]$} & {$[10.1,22.9]$} & {$[4.798,10.702]$} \\
\hline$[9.5,18.3]$ & {$[4.368,10.332]$} & {$[11.5,20.2]$} & {$[7.254,13.246]$} & {$[8.7,15.3]$} & {$[4.098,10.102]$} & {$[12.9,20.6]$} & {$[6.667,14.033]$} \\
\hline$[9.4,16.3]$ & {$[4.497,8.503]$} & {$[10.0,18.7]$} & {$[6.079,11.621]$} & {$[9.4,19.3]$} & {$[3.992,11.008]$} & {$[11.2,20.5]$} & {$[6.557,11.543]$} \\
\hline$[13.9,18.7]$ & {$[6.154,11.946]$} & {$[10.8,16.4]$} & {$[5.196,9.504]$} & {$[13.8,20.9]$} & {$[8.452,12.648]$} & {$[9.7,14.1]$} & {$[4.983,9.517]$} \\
\hline$[9.2,16.9]$ & {$[5.061,10.639]$} & {$[10.8,17.6]$} & {$[5.676,12.024]$} & {$[9.6,18.4]$} & {$[4.163,10.137]$} & {$[8.9,17.5]$} & {$[4.294,11.606]$} \\
\hline$[10.5,16.3]$ & {$[5.871,11.629]$} & {$[9.9,15.8]$} & {$[4.867,10.433]$} & {$[10.8,17.5]$} & {$[5.491,9.709]$} & {$[7.6,15.4]$} & {$[3.883,10.517]$} \\
\hline$[14.2,21.3]$ & {$[7.783,13.317]$} & {$[10.2,16.0]$} & {$[5.062,9.738]$} & {$[12.9,20.1]$} & {$[5.564,10.836]$} & {$[10.1,19.6]$} & {$[4.782,11.418]$} \\
\hline$[11.7,17.8]$ & {$[6.182,11.118]$} & {$[9.4,16.1]$} & {$[4.770,10.830]$} & {$[11.0,16.4]$} & {$[5.986,11.514]$} & {$[12.6,19.4]$} & {$[7.159,12.341]$} \\
\hline$[8.3,18.4]$ & {$[4.065,9.335$} & {$[10.9,17.2]$} & {$[4.893,9.107]$} & {$[11.7,18.9]$} & {$[5.573,10.827]$} & {$[12.0,17.9]$} & {$[7.279,11.421]$} \\
\hline$[10.2,19.5]$ & {$[4.282,9.918]$} & {$[10.3,16.2]$} & {$[6.278,10.322]$} & {$[9.6,16.5]$} & {$[4.962,10.138]$} & {$[9.6,18.4]$} & {$[4.294,9.206]$} \\
\hline$[9.8,16.0]$ & {$[3.898,9.002]$} & {$[9.8,17.8]$} & {$[4.962,12.038]$} & {$[11.5,19.9]$} & {$[4.754,10.946]$} & {$[10.9,21.6]$} & {$[5.156,11.144]$} \\
\hline$[13.0,20.6]$ & {$[6.174,11.726]$} & {$[8.7,18.0]$} & {$[4.167,10.233]$} & {$[14.7,20.7]$} & {$[8.659,12.841]$} & {$[13.3,20.9]$} & {$[6.864,13.836]$} \\
\hline$[11.6,20.0]$ & {$[5.664,11.236]$} & {$[12.6,18.4]$} & {$[6.354,11.846]$} & {$[10.9,19.4]$} & {$[4.958,9.642]$} & {$[12.7,19.5]$} & {$[7.566,12.134]$} \\
\hline$[10.9,15.9]$ & {$[6.074,11.626]$} & {$[11.1,17.3]$} & {$[5.792,10.408]$} & {$[10.2,16.9]$} & {$[5.878,10.722]$} & {$[9.5,15.9]$} & {$[5.955,10.145]$} \\
\hline$[11.6,19.0]$ & {$[5.564,9.936]$} & {$[8.3,16.4]$} & {$[3.797,11.203]$} & {$[10.1,20.0]$} & {$[5.177,12.923]$} & {$[12.3,20.5]$} & {$[5.268,10.232]$} \\
\hline$[9.2,18.0]$ & {$[4.786,8.914]$} & {$[8.5,14.5]$} & {$[4.158,8.642]$} & {$[8.8,16.0]$} & {$[3.656,8.444]$} & {$[10.4,16.0]$} & {$[5.099,11.201]$} \\
\hline$[10.3,17.0]$ & {$[5.662,10.538]$} & {$[12.8,26.2]$} & {$[6.893,16.207]$} & {$[8.0,14.9]$} & {$[3.481,8.219]$} & {$[9.6,17.9]$} & {$[4.863,8.537]$} \\
\hline$[13.4,22.5]$ & {$[6.597,12.403]$} & {$[9.2,14.7]$} & {$[4.466,10.334]$} & {$[9.7,17.4]$} & {$[3.773,8.427]$} & {$[10.4,16.0]$} & {$[6.151,10.949]$} \\
\hline$[11.3,17.8]$ & {$[5.773,9.727]$} & {$[8.9,17.6]$} & {$[4.200,10.500]$} & {$[9.8,20.2]$} & {$[5.278,12.122]$} & {$[12.3,18.3]$} & {$[5.871,10.029]$} \\
\hline$[10.2,18.4]$ & {$[4.071,8.429]$} & {$[8.3,15.4]$} & {$[4.289,8.311]$} & {$[8.6,18.3]$} & {$[3.781,9.319]$} & {$[10.4,19.7]$} & {$[4.578,9.822]$} \\
\hline$[9.7,17.1]$ & {$[5.583,10.717]$} & {$[13.6,26.1]$} & {$[7.176,15.424]$} & {$[9.6,17.5]$} & {$[3.855,8.445]$} & {$[13.0,18.8]$} & {$[6.976,12.724]$} \\
\hline$[10.8,18.9]$ & {$[5.500,11.000]$} & {$[10.8,17.5]$} & {$[5.865,11.435]$} & {$[10.9,18.5]$} & {$[5.554,10.246]$} & {$[12.8,16.9]$} & {$[6.794,10.806]$} \\
\hline
\end{tabular}

had been initially recorded as $Y^{I}$ in the hospital computer system. But then, $Y^{I}$ was recorded as $Y$ to match the decimal of $Y^{I}$ with the decimal of $X$.

In this study, we have $n=1, k=100$, and hence $n k=100$ (the realization of the first condition). We use the sample data for the interval variables $Y$ and $Y^{I}$, respectively, in Tables 5 and 4 . The $P$ - value $=0.177$ for the one sample Kolmogorov-Smirnov test at the significance level 0.05 shows that the values $G_{1}^{1 *}, \cdots, G_{100}^{1 *}$ are observations from the real-valued variable $G \sim \mathrm{U}\left(-0.049,0.049\right.$ ) (this means $G \stackrel{\mathcal{L}}{\longrightarrow} \underline{0}$, and hence $Y^{I}-Y \stackrel{\mathcal{L}}{\longrightarrow} \underline{0}$ (the second condition) is confirmed).

Based on the sample data for $(X, Y)$ in Table 5 , we obtain the values $0.5153,0.3212,0.5854$, and 1.4596, respectively, for the old known estimators $\widehat{\alpha}, \widehat{\beta}, \widehat{\gamma}$, and $\widehat{\delta}$ in Section 4.1 (or in Blanco-Fernández et al. , 2011). Also, the values $0.5153,0.3204,0.5854$, and 1.4898 , respectively, for the new estimators 
Table 5. Data set on the range of variation of blood pressures of 100 patients up to one decimal place.

\begin{tabular}{|c|c|c|c|c|c|c|c|}
\hline$X$ & $Y$ & $X$ & $Y$ & $X$ & $Y$ & $X$ & $Y$ \\
\hline$[15.0,20.5]$ & {$[7.4,12.5]$} & {$[8.6,14.5]$} & {$[4.7,9.1]$} & {$[13.8,19.9]$} & {$[4.6,9.1]$} & {$[13.2,17.9]$} & {$[5.5,9.5]$} \\
\hline$[10.5,20.8]$ & {$[6.2,11.5]$} & {$[9.0,17.0]$} & {$[4.4,11.3]$} & {$[8.2,16.5]$} & {$[3.6,9.3]$} & {$[10.0,21.6]$} & {$[4.3,10.2]$} \\
\hline$[11.5,20.1]$ & {$[6.7,13.1]$} & {$[8.5,16.8]$} & {$[4.2,10.0]$} & {$[9.3,15.5]$} & {$[5.5,10.6]$} & {$[8.5,17.2]$} & {$[3.7,8.4]$} \\
\hline$[11.3,17.0]$ & {$[5.6,10.7]$} & {$[11.4,18.1]$} & {$[4.0,8.8]$} & {$[12.6,17.6]$} & {$[5.2,9.6]$} & {$[10.1,22.9]$} & {$[4.8,10.7]$} \\
\hline$[9.5,18.3]$ & {$[4.4,10.3]$} & {$[11.5,20.2]$} & {$[7.3,13.2]$} & {$[8.7,15.3]$} & {$[4.1,10.1]$} & {$[12.9,20.6]$} & {$[6.7,14.0]$} \\
\hline$[9.4,16.3]$ & {$[4.5,8.5]$} & {$[10.0,18.7]$} & {$[6.1,11.6]$} & {$[9.4,19.3]$} & {$[4.0,11.0]$} & {$[11.2,20.5]$} & {$[6.6,11.5]$} \\
\hline$[13.9,18.7]$ & {$[6.2,11.9]$} & {$[10.8,16.4]$} & {$[5.2,9.5]$} & {$[13.8,20.9]$} & {$[8.5,12.6]$} & {$[9.7,14.1]$} & {$[5.0,9.5]$} \\
\hline$[9.2,16.9]$ & {$[5.1,10.6]$} & {$[10.8,17.6]$} & {$[5.7,12.0]$} & {$[9.6,18.4]$} & {$[4.2,10.1]$} & {$[8.9,17.5]$} & {$[4.3,11.6]$} \\
\hline$[10.5,16.3]$ & {$[5.9,11.6]$} & {$[9.9,15.8]$} & {$[4.9,10.4]$} & {$[10.8,17.5]$} & {$[5.5,9.7]$} & {$[7.6,15.4]$} & {$[3.9,10.5]$} \\
\hline$[14.2,21.3]$ & {$[7.8,13.3]$} & {$[10.2,16.0]$} & {$[5.1,9.7]$} & {$[12.9,20.1]$} & {$[5.6,10.8]$} & {$[10.1,19.6]$} & {$[4.8,11.4]$} \\
\hline$[11.7,17.8]$ & {$[6.2,11.1]$} & {$[9.4,16.1]$} & {$[4.8,10.8]$} & {$[11.0,16.4]$} & {$[6.0,11.5]$} & {$[12.6,19.4]$} & {$[7.2,12.3]$} \\
\hline$[8.3,18.4]$ & {$[4.1,9.3]$} & {$[10.9,17.2]$} & {$[4.9,9.1]$} & {$[11.7,18.9]$} & {$[5.6,10.8]$} & {$[12.0,17.9]$} & {$[7.3,11.4]$} \\
\hline$[10.2,19.5]$ & {$[4.3,9.9]$} & {$[10.3,16.2]$} & {$[6.3,10.3]$} & {$[9.6,16.5]$} & {$[5.0,10.1]$} & {$[9.6,18.4]$} & {$[4.3,9.2]$} \\
\hline$[9.8,16.0]$ & {$[3.9,9.0]$} & {$[9.8,17.8]$} & {$[5.0,12.0]$} & {$[11.5,19.9]$} & {$[4.8,10.9]$} & {$[10.9,21.6]$} & {$[5.2,11.1]$} \\
\hline$[13.0,20.6]$ & {$[6.2,11.7]$} & {$[8.7,18.0]$} & {$[4.2,10.2]$} & {$[14.7,20.7]$} & {$[8.7,12.8]$} & {$[13.3,20.9]$} & {$[6.9,13.8]$} \\
\hline$[11.6,20.0]$ & {$[5.7,11.2]$} & {$[12.6,18.4]$} & {$[6.4,11.8]$} & {$[10.9,19.4]$} & {$[5.0,9.6]$} & {$[12.7,19.5]$} & {$[7.6,12.1]$} \\
\hline$[10.9,15.9]$ & {$[6.1,11.6]$} & {$[11.1,17.3]$} & {$[5.8,10.4]$} & {$[10.2,16.9]$} & {$[5.9,10.7]$} & {$[9.5,15.9]$} & {$[6.0,10.1]$} \\
\hline$[11.6,19.0]$ & {$[5.6,9.9]$} & {$[8.3,16.4]$} & {$[3.8,11.2]$} & {$[10.1,20.0]$} & {$[5.2,12.9]$} & {$[12.3,20.5]$} & {$[5.3,10.2]$} \\
\hline$[9.2,18.0]$ & {$[4.8,8.9]$} & {$[8.5,14.5]$} & {$[4.2,8.6]$} & {$[8.8,16.0]$} & {$[3.7,8.4]$} & {$[10.4,16.0]$} & {$[5.1,11.2]$} \\
\hline$[10.3,17.0]$ & {$[5.7,10.5]$} & {$[12.8,26.2]$} & {$[6.9,16.2]$} & {$[8.0,14.9]$} & {$[3.5,8.2]$} & {$[9.6,17.9]$} & {$[4.9,8.5]$} \\
\hline$[13.4,22.5]$ & {$[6.6,12.4]$} & {$[9.2,14.7]$} & {$[4.5,10.3]$} & {$[9.7,17.4]$} & {$[3.8,8.4]$} & {$[10.4,16.0]$} & {$[6.2,10.9]$} \\
\hline$[11.3,17.8]$ & {$[5.8,9.7]$} & {$[8.9,17.6]$} & {$[4.2,10.5]$} & {$[9.8,20.2]$} & {$[5.3,12.1]$} & {$[12.3,18.3]$} & {$[5.9,10.0]$} \\
\hline$[10.2,18.4]$ & {$[4.1,8.4]$} & {$[8.3,15.4]$} & {$[4.3,8.3]$} & {$[8.6,18.3]$} & {$[3.8,9.3]$} & {$[10.4,19.7]$} & {$[4.6,9.8]$} \\
\hline$[9.7,17.1]$ & {$[5.6,10.7]$} & {$[13.6,26.1]$} & {$[7.2,15.4]$} & {$[9.6,17.5]$} & {$[3.9,8.4]$} & {$[13.0,18.8]$} & {$[7.0,12.7]$} \\
\hline$[10.8,18.9]$ & {$[5.5,11.0]$} & {$[10.8,17.5]$} & {$[5.9,11.4]$} & {$[10.9,18.5]$} & {$[5.6,10.2]$} & {$[12.8,16.9]$} & {$[6.8,10.8]$} \\
\hline
\end{tabular}


$\widehat{\alpha}, \widehat{\beta}, \widehat{\gamma}$, and $\widehat{\delta}$ in Section 4.2 are calculated using the sample data for $\left(X, Y^{I}\right)$ in Table 4. By considering $\left(\frac{M S E M N}{M S E M O}\right)=\left(\frac{0.9172}{0.9186}\right)=0.9984<1$, the new estimation method of the model (1) in Section 4.2 is superior to the old known estimation method of the model in Section 4.1.

Based on the sample data for $(X, Y)$ and $\left(X, Y^{I}\right)$, respectively, in Tables 5 and 4, the existence of the assumptions of theorems is confirmed as follows: First, the first and second conditions are confirmed $(n k=100$ and $\left.Y^{I}-Y \stackrel{\mathcal{L}}{\longrightarrow} \underline{0}\right)$. Second, we obtain $\widehat{\sigma}^{2}(X)=3.8752, \widehat{\sigma}^{2}\left(X^{M}\right)=3.0664$, and $\widehat{\sigma}^{2}\left(X^{S}\right)=0.8089$. Third, we have $\widehat{\sigma}^{2}(Y)=1.8257, \widehat{\sigma}^{2}\left(Y^{M}\right)=1.5555$, $\widehat{\sigma}^{2}\left(Y^{S}\right)=0.2702$. Fourth, $\widehat{\sigma}^{2}\left(Y^{I}\right)=1.8238, \widehat{\sigma}^{2}\left(Y^{I M}\right)=1.5555$, and $\widehat{\sigma}^{2}\left(Y^{I S}\right)=0.2683$ are obtained. Fifth, $\widehat{\sigma}(X, Y)=1.8400, \widehat{\sigma}\left(X^{M}, Y^{M}\right)=$ 1.5802, $\widehat{\sigma}\left(X^{S}, Y^{S}\right)=0.2598, \widehat{\sigma}\left(X, Y^{I}\right)=1.8393, \widehat{\sigma}\left(X^{M}, Y^{I M}\right)=1.5802$, and $\widehat{\sigma}\left(X^{S}, Y^{I S}\right)=0.2591$ are calculated. Sixth, we have $\widehat{\sigma}\left(X^{S}, Y^{M}\right)=$ $2.0056 e-7, \widehat{\sigma}\left(X^{M}, Y^{S}\right)=1.5981 e-9, \widehat{\sigma}\left(X^{S}, Y^{I M}\right)=2.0056 e-7$, and $\widehat{\sigma}\left(X^{M}, Y^{I S}\right)=1.5961 e-9$. Seventh, we know $\widehat{\varepsilon}=\widehat{\varepsilon}^{M}+\widehat{\varepsilon}^{S}$, mid $\widehat{\varepsilon}=$ $\operatorname{mid} Y-\operatorname{mid} \widehat{Y}$, and $\operatorname{spr} \widehat{\varepsilon}=\operatorname{spr} Y-\operatorname{spr} \widehat{Y}$, where $\operatorname{mid} \widehat{Y}=\widehat{\alpha} \operatorname{mid} X+\widehat{\gamma}$ and $\operatorname{spr} \widehat{Y}=\widehat{\beta} \operatorname{spr} X+\widehat{\delta}$. Hence, considering Section 2.1, $\widehat{\sigma}^{2}(\varepsilon)=0.9279$, $\widehat{\sigma}^{2}\left(\varepsilon^{M}\right)=0.7412, \widehat{\sigma}^{2}\left(\varepsilon^{S}\right)=0.1867, \widehat{\sigma}(X, \varepsilon)=-1.5168 e-13, \widehat{\sigma}\left(X^{M}, \varepsilon^{M}\right)=$ $-1.4846 e-13, \widehat{\sigma}\left(X^{S}, \varepsilon^{S}\right)=-3.2191 e-15$ are obtained. Eighth, as results from the seventh and first cases, we conclude $\frac{\widehat{\sigma}^{2}\left(\varepsilon^{M}\right)}{\widehat{\sigma}^{2}\left(X^{M}\right)}=0.2417$ and $\frac{\widehat{\sigma}^{2}\left(\varepsilon^{S}\right)}{\widehat{\sigma}^{2}\left(X^{S}\right)}=0.2308$.

\section{Conclusions}

In many recent articles, the relationships between interval-valued variables have been modeled by LRMs. If interval response variables have any statistical distributions, the relationships are modeled in the LMs framework.

The new structure of MCR in Section 3 randomly generates a large number of single-valued sample sets (the sets consist of points randomly chosen within random intervals of the interval variables). Hence, the structure is computationally intensive. However, this disadvantage is outweighed by its good properties. First, the internal variations of intervals are fully utilized using the structure. Second, it relieves the need to develop complex methodologies for interval-valued data. Third, it is flexible in the sense that one can modify the structure of MCR depending on specific problems. For example, one may assume a non-uniform distribution within an interval such 
as truncated normal distribution.

Using the new structure of MCR, we introduce the set of new estimators (see Section 4.2) alongside the set of old known estimators (see Section 4.1) for the set of parameters of the model (1) under the conditions. In this work, under the conditions, the theoretical performance of each of the old known and new estimators is shown. We study the asymptotic unbiasedness, the strong consistency, the asymptotic distribution, the asymptotic variance, and the asymptotic MSE of each estimator under the conditions. Also, in each mentioned asymptotic property, each new estimator is compared with the corresponding old known estimator.

Under the conditions, the results of the simulations in Section 6.1 show the empirical performance (the empirical asymptotic unbiasedness and the empirical consistency) of the old known and new estimators of the model (1). The assessments in Section 6.1 demonstrate well that for LMs with the structure of (1), the distributions of the variables $\operatorname{spr} \varepsilon$ and $\operatorname{spr} X$ are simultaneously effective in choosing a more efficient set of estimators from among the two sets under the conditions. Under the conditions, the new estimators are superior to the old known estimators for estimating the parameters of some LMs with the structure of (1). Under the conditions, Table 3 shows no absolute superiority of one of the sets of the old known and new estimators to another.

\section{Acknowledgment}

The authors are thankful to the referees for their valuable comments.

\section{References}

Afonso, F., Billard, L., Diday, E. and Limam, M. (2007). Symbolic Linear Regression Methodology. Symbolic Data Analysis and the SODAS Software, 359-372.

Artstein, Z. and Vitale, R.A. (1975). A Strong Law of Large Numbers for Random Compact Sets. Ann. Probability, 5, 879-882.

Bertrand, P. and Goupil, F. (2000). Descriptive Statistics for Symbolic Data. In Bock, H.H. and Diday, E. (eds) Analysis of Symbolic Data (pp. 106-124). Studies in Classification, Data Analysis, and Knowledge Organization. Springer Berlin Heidelberg.

Billard, L. (2007). Dependencies and Variation Components of Symbolic Interval-valued Data. In Brito, P., Cucumel, G., Bertrand, P. and de Carvalho, F. (eds) Selected Contributions in 
Data Analysis and Classification (pp. 3-12). Studies in Classification, Data Analysis, and Knowledge Organization. Springer Berlin Heidelberg.

Billard, L. (2011). Brief Overview of Symbolic Data and Analytic Issues. Stat. Anal. Data Min., 4, 149-156.

Billard, L. and Diday, E. (2000). Regression Analysis for Interval-valued Data. In Kiers, H.A.L., Rasson, J.P., Groenen, P.J.F. and Schader, M. (eds) Data Analysis, Classification, and Related Methods (pp. 369-374). Studies in Classification, Data Analysis, and Knowledge Organization. Springer Berlin Heidelberg.

Blanco-Fernández, A., Corral, N. and González-Rodríguez, G. (2011). Estimation of a Flexible Simple Linear Model for Interval Data Based on Set Arithmetic. Comput. Statist. Data Anal., 55, 2568-2578.

Calle, M.L. and Gómez, G. (2001). Nonparametric Bayesian Estimation from Intervalcensored Data Using Monte Carlo Methods. J. Statist. Plann. Inference, 98, 73-87.

Diday, E. (1995). Probabilist, Possibilist and Belief Objects for Knowledge Analysis. Annal. Operat. Research, 55, 225-276.

Diday, E. and Émilion, R. (1998). Capacities, Credibilities in Analysis of Probabilistic Objects by Histograms and Lattices. In Hayashi, C., Yajima, K., Bock, H.H., Ohsumi, N., Tanaka, Y. and Baba, Y. (eds) Data Science, Classification, and Related Methods (pp. 353-357). Studies in Classification, Data Analysis, and Knowledge Organization. Springer Tokyo.

Émilion, R. (1997). Différentiation des Capacités et des Intégrales de Choquet. Comptes Rendus de l'Académie des Sciences. Mathématique, 324, 389-392.

Gil, M.Á., González-Rodríguez, G., Colubi, A. and Montenegro, M. (2007). Testing Linear Independence in Linear Models with Interval-valued Data. Comput. Statist. Data Anal., 51, 3002-3015.

Huber, C., Solev, V. and Vonta, F. (2009). Interval Censored and Truncated Data: Rate of Convergence of NPMLE of the Density. J. Statist. Plann. Inference, 139, 1734-1749.

Kim, J. and Billard, L. (2011). A Polythetic Clustering Process and Cluster Validity Indexes for Histogram-valued Objects. Comput. Statist. Data Anal., 55, 2250-2262.

Le-Rademacher, J. and Billard, L. (2012). Symbolic Covariance Principal Component Analysis and Visualization for Interval-valued Data. J. Comput. Graph. Statist., 21, 413-432.

Neto, E.A.L. and de Carvalho, F.A.T. (2008). Centre and Range Method for Fitting a Linear Regression Model to Symbolic Interval Data. Comput. Statist. Data Anal., 52, 1500-1515.

Neto, E.A.L. and de Carvalho, F.A.T. (2010). Constrained Linear Regression Models for Symbolic Interval-valued Variables. Comput. Statist. Data Anal., 54, 333-347.

J. Statist. Res. Iran 15 (2018): 237-274 
Neto, E.A.L., de Carvalho, F.A.T. and Tenorio, C. P. (2004). Univariate and Multivariate Linear Regression Methods to Predict Interval-valued Features. In The Australasian Joint Conference on Artificial Intelligence (pp. 526-537). Springer Berlin Heidelberg.

Neto, E.A.L., de Carvalho, F.A.T. and Freire, E.S. (2005). Applying Constrained Linear Regression Models to Predict Interval-valued Data. In The Annual Conference on Artificial Intelligence (pp. 92-106). Springer Brelin Heidelberg.

Rivero, C. and Valdes, T. (2008). An Algorithm for Robust Linear Estimation with Grouped Data. Comput. Statist. Data Anal., 53, 255-271.

Trutschnig, W., González-Rodríguez, G., Colubi, A. and Gil, M.Á. (2009). A New Family of Metrics for Compact, Convex (Fuzzy) Sets Based on a Generalized Concept of Mid and Spread. Inform. Sci., 179, 3964-3972.

Wang, H., Guan, R. and Wu, J. (2012). Linear Regression of Interval-valued Data Based on Complete Information in Hypercubes. J. Syst. Sci. Syst. Eng., 21, 422-442.

\section{Amir Massoud Malekfar}

Department of Statistics,

Allameh Tabataba'i University,

Tehran, Iran.

email:malekfar1364@gmail.com

\section{Farzad Eskandari}

Department of Statistics,

Allameh Tabataba'i University,

Tehran, Iran.

email: askandari@atu.ac.ir 